\title{
Modeling Financial Time Series Based on a Market Microstructure Model with Leverage Effect
}

\author{
Yanhui Xi, ${ }^{1,2,3}$ Hui Peng, ${ }^{2,3}$ and Yemei Qin ${ }^{2,3}$ \\ ${ }^{1}$ Hunan Province Higher Education Key Laboratory of Power System Safety Operation and Control, \\ Changsha University of Science and Technology, Changsha, Hunan 410004, China \\ ${ }^{2}$ School of Information Science \& Engineering, Central South University, Changsha, Hunan 410083, China \\ ${ }^{3}$ Hunan Engineering Laboratory for Advanced Control and Intelligent Automation, Changsha, Hunan 410083, China
}

Correspondence should be addressed to Hui Peng; huipeng@mail.csu.edu.cn

Received 31 August 2015; Accepted 21 December 2015

Academic Editor: Filippo Cacace

Copyright (C) 2016 Yanhui Xi et al. This is an open access article distributed under the Creative Commons Attribution License, which permits unrestricted use, distribution, and reproduction in any medium, provided the original work is properly cited.

\begin{abstract}
The basic market microstructure model specifies that the price/return innovation and the volatility innovation are independent Gaussian white noise processes. However, the financial leverage effect has been found to be statistically significant in many financial time series. In this paper, a novel market microstructure model with leverage effects is proposed. The model specification assumed a negative correlation in the errors between the price/return innovation and the volatility innovation. With the new representations, a theoretical explanation of leverage effect is provided. Simulated data and daily stock market indices (Shanghai composite index, Shenzhen component index, and Standard and Poor's 500 Composite index) via Bayesian Markov Chain Monte Carlo (MCMC) method are used to estimate the leverage market microstructure model. The results verify the effectiveness of the model and its estimation approach proposed in the paper and also indicate that the stock markets have strong leverage effects. Compared with the classical leverage stochastic volatility (SV) model in terms of DIC (Deviance Information Criterion), the leverage market microstructure model fits the data better.
\end{abstract}

\section{Introduction}

Financial system is a complex dynamic system. Many mathematical models have already been proposed to describe the dynamics of financial markets. With the fast development of mathematical finance in recent years, stochastic differential equations have been widely used to describe the dynamics of a wide variety of random in finance. The current most acceptable theorem of price movements in financial markets is that they are random walks with predictive errors close to white noise and this is due to the Markov properties of financial time series. Hence, much of financial theory is based on this assumption.

It has been long recognized that the returns of financial assets are negatively correlated with changes in the volatilities of returns; that is, there is leverage effect between volatility and price/return. Black [1] and Christie [2] have found empirical evidence of the leverage effect; that is, volatility tends to rise in response to bad news but fall in response to good news. Christie [2] provides a theoretical explanation of leverage effect under a Modigliani/Miller economy.

Modeling of financial market volatility has been one of the most active areas of research in empirical finance and time series econometrics over the past two decades. Two types of volatility models, the ARCH (autoregressive conditional heteroskedasticity) model and the SV (stochastic volatility) model, are well estimated in financial econometrics. Numerous researchers develop their extensions and found overwhelming evidence of leverage effect. For instance, Bollerslev [3] proposed the generalized ARCH (GARCH) model, which formulates the serial dependence of volatility and incorporates the past observations into the future volatility. Nelson [4] proposed the EGARCH (exponential GARCH) specification, modeling the leverage effect, which refers to the increase in volatility following a previous drop in stock returns. while Glosten et al. [5] developed a threshold indicator function GARCH model with leverage effect, which is commonly called the GJR model. A common idea used 
in the above models is the leverage effect, in which negative shocks to price/return increase the predictable volatility to a greater extent than do positive shocks.

On the other hand, regarding volatility clustering, the stochastic volatility (SV) model has been widely used to model the time-varying variance of time series in financial econometrics. In the SV literature, the asymmetric (leverage) volatility response is often studied by specifying a negative correlation between the return innovation and the volatility innovation. This classical leverage SV model, proposed by Harvey and Shephard [6], requires correlation between the return innovation $\xi_{t}$ and the volatility innovation $\eta_{t+1}$. Instead of the dependence, that is, $\operatorname{corr}\left(\xi_{t}, \eta_{t+1}\right)=\rho$, Jacquier et al. [7] generalized the basic SV model with leverage effect by allowing for an dependence between $\xi_{t}$ and $\eta_{t}$; that is, $\operatorname{corr}\left(\xi_{t}, \eta_{t}\right)=\rho$. Yu [8] gives comments on the JPR specification [7] and shows that it does not necessarily lead to a leverage effect and hence is not theoretically justified. Following Harvey and Shephard [6], Jacquier et al. [7], Yu [8, 9], Omori et al. [10], Wang et al. [11], Asai and McAleer [12], and Tsiotas [13] extend the SV specification with leverage.

Differing from the ARCH model and the SV model, a phenomenological model based on identifying the processes influencing the demand and supply of a market was proposed, which was called the market microstructure model [14]. O'Hara [15] defines market microstructure as "the study of the process and outcomes of exchanging assets under a specific set of rules. While much of economics abstracts from the mechanics of trading, microstructure theory focuses on how specific trading mechanisms affect the price formation process." In microeconomics, supply and demand is an economic model of price determination in a market; see, for example, Marshall [16], Zhang [17]. On the other hand, the market liquidity affects the asset prices and expected returns. Theory and empirical evidence suggests that, for an asset with given cash flow, the higher its market liquidity, the lower its expected return (e.g., $[18,19])$. In this paper, the market microstructure model assumes that asset price is driven by the excess demand, and the amplitude of price changes is dependent on the liquidity of the market. Some improvements of the market microstructure model and their estimation approaches as well as applications $[20,21]$ were also presented. Although Peng et al. [22, 23] and Xi et al. [24, 25] proposed the generalized market microstructure (GMMS) models, which included jump component for capturing the low-frequency and large-amplitude abnormal vibrations of price, they did not consider the important property, namely, leverage effect. Recently, to explain essential characteristics of skewness and heavy tails, $\mathrm{Xi}$ et al. [25] proposed the heavy-tailed market microstructure model based on Student$t$ distribution (MM- $t$ ). However, all of the above market microstructure models are difficult to explain the asymmetry in the relation between volatility and price/return. Motivated by the empirical evidence, this paper is concerned with the specification for modeling financial leverage effect in the market microstructure model, which allows for a dependence between the price innovation and the volatility innovation to pick up the kind of leverage behavior.
The remainder of the study is organized as follows. Section 2 introduces the market microstructure model with leverage and gives a theoretical explanation of leverage effect. In Section 3, we discuss the MCMC estimation for our market microstructure model with leverage. Section 4 describes the simulated data results by MCMC method. Section 5 displays and discusses the empirical findings using the leverage market microstructure model. Section 6 provides the model comparison between the leverage SV model and the leverage market microstructure model. Section 7 gives some concluding remarks.

\section{Leverage in the Market Microstructure Model}

To deal with the dynamics of a financial market from different points of view, one phenomenological microstructure model based on identifying different processes influencing the demand and supply of the market is defined by [26]

$$
d P_{t}=\lambda \phi_{t} d t
$$

where $P_{t}$ is the asset price, $\lambda$ is the (inverse of) market liquidity, and $\phi_{t}$ is the excess demand which is defined by $\phi_{t}=\phi_{t}^{+}-\phi_{t}^{-}$, where $\phi_{t}^{+}$is the instantaneous demand and $\phi_{t}^{-}$is the instantaneous supply at any given instant of time for the asset. $\phi_{t}$ characterizes whether the market is overvalued $\left(\phi_{t}>0\right.$, which tends to push the price up) or undervalued ( $\phi_{t}<0$, which tends to push the price down). Model (1) assumes that price $P_{t}$ is driven by the excess demand $\phi_{t}$, and the amplitude of price changes is determined by the market liquidity, that is, $1 / \lambda$.

However, model (1) only provides an abstract description for the dynamics of market. To estimate the two hidden market state variables $\left(\lambda\right.$ and $\left.\phi_{t}\right)$ from a time series of price, model (1) can then be written as $[14,20]$

$$
\begin{aligned}
d P_{t} & =\lambda_{t} \phi_{t} d t+\lambda_{t} d w_{1, t}, \\
d \phi_{t} & =\left(\alpha_{1}+\beta_{1} \phi_{t}\right) d t+\gamma_{1} d w_{2, t}, \\
d \log \lambda_{t} & =\left(\alpha_{2}+\beta_{2} \log \lambda_{t}\right) d t+\gamma_{2} d w_{3, t},
\end{aligned}
$$

where $w_{1, t}, w_{2, t}$, and $w_{3, t}$ are independent Brownian motion processes and $\alpha_{1}, \beta_{1}, \alpha_{2}, \beta_{2}$, and $\gamma_{1}$ and $\gamma_{2}$ are constant parameters.

The first equation in model (2) describes the financial asset price process. It is notable that the conditional expected value and the conditional variance of the financial asset price process are given by

$$
\begin{aligned}
\mathrm{E}\left(d P_{t}\right) & =\mathrm{E}\left(\lambda_{t} \phi_{t} d t+\lambda_{t} d w_{1, t}\right)=\lambda_{t} \phi_{t} d t, \\
\operatorname{Var}\left(d P_{t}\right) & =\operatorname{Var}\left(\lambda_{t} \phi_{t} d t+\lambda_{t} d w_{1, t}\right)=\lambda_{t}^{2} d t .
\end{aligned}
$$

The second equation in model (2) models the process of the immeasurable hidden excess demand variable $\phi_{t}$. The third equation in model (2) models the dynamics of liquidity, which together with the first equation resembles a stochastic volatility (SV) model in which the variance is specified to 
follow some latent stochastic process. Consequently, the third equation reveals characterization of volatility dynamics. In contrast with the SV model and the ARCH model, the market microstructure model (model (2)) offers a better representation of the internal characteristics of a financial price varying process and information which possess better stability than the market trend information obtained for the mere prediction of a price process.

To make the above market microstructure model characterize the leverage effect for financial variables, here we assume that $w_{1, t}, w_{3, t}$ are two dependence Brownian motions with correlation measured by $\rho$; that is, $\operatorname{corr}\left(d w_{1, t}, d w_{3, t}\right)=\rho$. Here, the correlation coefficient $\rho<0$ implies model (2) with leverage effect, while $\rho=0$ implies model (2) without leverage effect.

In the empirical literature, the continuous model is often discretized to facilitate estimation. In mathematics, the EulerMaruyama method [27] is a simple and generalized method for the approximate numerical solution of a stochastic differential equation.

A general form for a stochastic differential equation is

$$
\begin{aligned}
d X_{t} & =a\left(X_{t}\right) d t+b\left(X_{t}\right) d w_{t}, \\
X(0) & =X_{0},
\end{aligned}
$$

where $w_{t}$ stands for the Wiener process that depends continuously on $t \in[0, T]$.

Stochastic differential equation (4) can be written in integral form as

$$
X_{t}=X_{0}+\int_{0}^{t} a\left(X_{s}\right) d s+\int_{0}^{t} b\left(X_{s}\right) d w_{s} .
$$

To construct a numerical method from the integral form (5), we begin by setting $\Delta t=T / N$ for some positive integer $N$ and $t_{n}=n \Delta t(n=1,2, \ldots, N)$ and also define the numerical approximation to $X\left(t_{n}\right)$ as $X_{n}$. Setting successively $t=t_{n+1}$ and $t=t_{n}$ in integral form (5), we can obtain

$$
\begin{gathered}
X\left(t_{n+1}\right)=X_{0}+\int_{0}^{t_{n+1}} a\left(X_{s}\right) d s+\int_{0}^{t_{n+1}} b\left(X_{s}\right) d w_{s} \\
X\left(t_{n}\right)=X_{0}+\int_{0}^{t_{n}} a\left(X_{s}\right) d s+\int_{0}^{t_{n}} b\left(X_{s}\right) d w_{s} .
\end{gathered}
$$

If we subtract (7) from (6), then we obtain

$$
X\left(t_{n+1}\right)=X\left(t_{n}\right)+\int_{t_{n}}^{t_{n+1}} a\left(X_{s}\right) d s+\int_{t_{n}}^{t_{n+1}} b\left(X_{s}\right) d w_{s} .
$$

We can now consider approximating each of the integral terms. For the first integral in (8), we can use the conventional deterministic quadrature:

$$
\int_{t_{n}}^{t_{n+1}} a\left(X_{s}\right) d s \approx\left(t_{n+1}-t_{n}\right) a\left(X_{n}\right)=\Delta t a\left(X_{n}\right) .
$$

And, for the second integral, we use the Ito formula:

$$
\int_{t_{n}}^{t_{n+1}} b\left(X_{s}\right) d w_{s} \approx b\left(X_{n}\right)\left(w_{n+1}-w_{n}\right)=\Delta w_{n} b\left(X_{n}\right) .
$$

By combining these together, the Euler-Maruyama method takes the form

$$
X_{n+1}=X_{n}+\Delta t a\left(X_{n}\right)+\Delta w_{n} b\left(X_{n}\right)
$$

where $\Delta w_{n}=\sqrt{\Delta t} \varepsilon(\varepsilon \sim N(0,1))$.

Using the above Euler-Maruyama discrete time approximation, model (2) can be then derived as follows:

$$
\begin{aligned}
P_{k} & =P_{k-1}+\phi_{k-1} \lambda_{k-1}+\gamma_{3} \lambda_{k-1} \xi_{1, k}, \\
\phi_{k} & =\alpha_{1}+\left(1+\beta_{1}\right) \phi_{k-1}+\gamma_{1} \xi_{2, k}, \\
\log \lambda_{k}^{2} & =\alpha_{2}+\left(1+\beta_{2}\right) \log \lambda_{k-1}^{2}+\gamma_{2} \xi_{3, k},
\end{aligned}
$$

where $\xi_{1, k}, \xi_{2, k}$, and $\xi_{3, k}$ are iid $N(0,1)$ (the abbreviation iid refers to independent and identically distributed) and $\xi_{1, k}$, $\xi_{3, k+1}$ are dependent errors with correlation measured by $\rho$; that is, $\operatorname{corr}\left(\xi_{1, k}, \xi_{3, k+1}\right)=\rho \cdot \gamma_{3}$ is a new constant parameter, which describes the relation between $P_{k}, \lambda_{k-1}$ and $\phi_{k-1}$.

To build the observation equation, $P_{k}$ is chosen as observation variable. So, the state space representation of model (12) is given by

$$
\begin{aligned}
\mathbf{X}_{k} & =\left(\begin{array}{c}
P_{k} \\
\phi_{k} \\
\log \lambda_{k}^{2}
\end{array}\right) \\
& =\left(\begin{array}{c}
P_{k-1}+\phi_{k-1} \lambda_{k-1}+\gamma_{3} \lambda_{k-1} \xi_{1, k} \\
\alpha_{1}+\left(1+\beta_{1}\right) \phi_{k-1}+\gamma_{1} \xi_{2, k} \\
\alpha_{2}+\left(1+\beta_{2}\right) \log \lambda_{k-1}^{2}+\gamma_{2} \xi_{3, k}
\end{array}\right), \\
Y_{k} & =P_{k}+\varepsilon_{1},
\end{aligned}
$$

where $\varepsilon_{1}$ is iid $N\left(0, e_{1}^{2}\right)$.

To fully understand the leverage effect, it is convenient to adopt the Gaussian nonlinear state space form with uncorrelated error terms. To do so, let us denote $\xi_{k+1} \equiv$ $\left(\xi_{3, k+1}-\rho \xi_{1, k}\right) / \sqrt{1-\rho^{2}}$ and rewrite (12) as

$$
\left.\begin{array}{l}
\left(\begin{array}{c}
P_{k} \\
\phi_{k} \\
\log \lambda_{k+1}^{2}
\end{array}\right) \\
=\left(\begin{array}{c}
P_{k-1}+\phi_{k-1} \lambda_{k-1}+\gamma_{3} \lambda_{k-1} \xi_{1, k} \\
\alpha_{1}+\left(1+\beta_{1}\right) \phi_{k-1}+\gamma_{1} \xi_{2, k} \\
\alpha_{2}+\left(1+\beta_{2}\right) \log \lambda_{k}^{2}+\gamma_{2}\left(\rho \xi_{1, k}+\sqrt{1-\rho^{2}} \xi_{k+1}\right.
\end{array}\right)
\end{array}\right),
$$

where $\xi_{k+1}$ is iid $N(0,1)$ and $\xi_{1, k}, \xi_{2, k}$, and $\xi_{k+1}$ are independent Gaussian white noise processes. 
Also, the system of (14) can be alternatively specified by

$$
\left(\begin{array}{c}
P_{k} \\
\phi_{k} \\
\log \lambda_{k+1}^{2}
\end{array}\right)=\left(\begin{array}{c}
P_{k-1}+\phi_{k-1} \lambda_{k-1}+\gamma_{3} \lambda_{k-1} \xi_{1, k} \\
\alpha_{1}+\left(1+\beta_{1}\right) \phi_{k-1}+\gamma_{1} \xi_{2, k} \\
\alpha_{2}+\left(1+\beta_{2}\right) \log \lambda_{k}^{2}+\frac{\gamma_{2} \rho\left(P_{k}-P_{k-1}-\phi_{k-1} \log \lambda_{k-1}^{2}\right)}{\left(\gamma_{3} \log \lambda_{k-1}^{2}\right)}+\gamma_{2} \sqrt{1-\rho^{2}} \xi_{k+1}
\end{array}\right)
$$

For simplicity, we set

$$
\sigma_{k}=\log \lambda_{k}^{2}
$$

In finance, the volatility of asset price corresponds to standard deviation. Consequently, $\sigma_{k}$ in (16) represents the volatility of asset price, which is explained by the liquidity process. define

To compute the partial derivative $\partial \sigma_{k+1} / \partial r_{k}$, we first

$$
\begin{aligned}
F\left(\sigma_{k+1}, r_{k}\right) \equiv & \sigma_{k+1}-\alpha_{2}-\left(1+\beta_{2}\right) \sigma_{k} \\
& -\frac{\gamma_{2} \rho\left(P_{k}-P_{k-1}-\phi_{k-1} e^{\sigma_{k-1} / 2}\right) e^{-\sigma_{k-1} / 2}}{\gamma_{3}} \\
& -\gamma_{2} \sqrt{1-\rho^{2}} \xi_{k+1} \\
= & \sigma_{k+1}-\alpha_{2}-\left(1+\beta_{2}\right) \sigma_{k} \\
& -\frac{\gamma_{2} \rho\left(r_{k}-\phi_{k-1} e^{\sigma_{k-1} / 2}\right) e^{-\sigma_{k-1} / 2}}{\gamma_{3}} \\
& -\gamma_{2} \sqrt{1-\rho^{2}} \xi_{k+1}=0,
\end{aligned}
$$

where $r_{k}=P_{k}-P_{k-1}$ is a compounded return. we have

According to the derivation rule of the implicit function,

$$
\frac{\partial \sigma_{k+1}}{\partial r_{k}}=-\frac{\partial F / \partial r_{k}}{\partial F / \partial \sigma_{k+1}}=\frac{\gamma_{2}}{\gamma_{3}} \rho e^{-\sigma_{k-1} / 2}
$$

Obviously, if $\rho<0$ and holding everything else constant, a fall in the stock return $\left(r_{k}<0\right)$ leads to an increase of future expected volatility $\sigma_{k+1}$. Instead, a rise in the stock return $\left(r_{k}>0\right)$ leads to an decrease of future expected volatility $\sigma_{k+1}$. This is a so-called leverage effect, a negative shock to returns that increases the predictable volatility to a greater extent than does a positive shock.

\section{Estimation of the Leverage Market Microstructure Model}

For estimation of the basic market microstructure model, Peng et al. [20] proposed the extended Kalman filter (EKF) and the maximum likelihood method. However, its state equations are highly nonlinear, the EKF may give a high estimation error, and it may lead to inaccurate maximum likelihood estimates. Recently, Markov Chain Monte Carlo (MCMC) methods have become one of the most important tools for estimating stochastic volatility models since it was introduced in Jacquier et al. [28] to analyze the basic SV model. A fast and reliable MCMC algorithm, proposed by Kim et al. [29], was used in the SV model (see e.g., [10, 30, 31]). On the other hand, Bauwens and Lubrano [32], Vrontos et al. [33], and Nakatsuma [34] develop the MCMC estimation method for the models in the GARCH class. Their estimation results are very good since MCMC provides a fully likelihood-based inference.

In statistics, Markov Chain Monte Carlo (MCMC) methods are a class of algorithms for sampling from probability distributions based on constructing a Markov chain that has the desired distribution as its equilibrium distribution. The state of the chain after a large number of steps is then used as a sample of the desired distribution. To facilitate an efficient posterior inference using WinBUGS (Windows version of Bayesian Analysis Using Gibbs Sampler, a statistical software for Bayesian analysis using MCMC methods. WinBUGS Homepage: http:/www.mrc-bsu.cam.ac.uk/bugs/ winbugs/contents.shtml), the following state and observation equations for the model (13) were obtained as

$$
\begin{aligned}
& \sigma_{k+1} \mid \sigma_{k}, \alpha_{2}, \beta_{2}, \gamma_{2} \sim N\left(\alpha_{2}+\left(1+\beta_{2}\right) \sigma_{k}, \gamma_{2}^{2}\right), \\
& \phi_{k} \mid \phi_{k-1}, \alpha_{1}, \beta_{1}, \gamma_{1} \sim N\left(\alpha_{1}+\left(1+\beta_{1}\right) \phi_{k-1}, \gamma_{1}^{2}\right), \\
& P_{k} \mid P_{k-1}, \phi_{k-1}, \sigma_{k+1}, \sigma_{k}, \sigma_{k-1}, \alpha_{1}, \beta_{1}, \alpha_{2}, \beta_{2}, \gamma_{1}, \gamma_{2}, \gamma_{3}, e_{1}^{2}, \rho \\
& \sim N\left(P_{k-1}+\phi_{k-1} e^{\sigma_{k-1} / 2}+\frac{\gamma_{3}}{\gamma_{2}}\right. \\
& \cdot \rho e^{\sigma_{k-1} / 2}\left(\sigma_{k+1}-\alpha_{2}-\left(1+\beta_{2}\right) \sigma_{k}\right), \\
& \left.\gamma_{3}^{2} e^{\sigma_{k-1}}\left(1-\rho^{2}\right)\right), \\
& Y_{k} \mid P_{k}, e_{1}^{2} \sim N\left(P_{k}, e_{1}^{2}\right) .
\end{aligned}
$$

In this context, we face the problem of simulating a multivariate density consisting of the parameter $\boldsymbol{\theta}=$ $\left(\alpha_{1}, \beta_{1}, \alpha_{2}, \beta_{2}, \gamma_{1}, \gamma_{2}, \gamma_{3}, e_{1}, \rho\right)$ and the unobserved states $P_{k}$, $\phi_{k}$, and $\sigma_{k}$. Let $Y_{1: K}=\left\{Y_{1}, \ldots, Y_{K}\right\}$ be the observed asset and the vectors $\mathbf{X}_{0: K}=\left\{P_{0: K}^{T}, \phi_{0: K}^{T}, \sigma_{0: K}^{T}\right\}^{T}$ are unobserved states, where $P_{0: K}=\left\{P_{0}, P_{1}, \ldots, P_{K}\right\}, \phi_{0: K}=\left\{\phi_{0}, \phi_{1}, \ldots, \phi_{K}\right\}$, and $\sigma_{0: K}=\left\{\sigma_{0}, \sigma_{1}, \ldots, \sigma_{K}\right\}$. By Bayes' theorem, the joint posterior 
distribution of the unobservable variables given the data is obtained as

$$
\begin{aligned}
& p\left(\alpha_{1}, \beta_{1}, \alpha_{2}, \beta_{2}, \gamma_{1}, \gamma_{2}, \gamma_{3}, e_{1}, \rho, P_{0: K}, \phi_{0: K}, \sigma_{0: K} \mid Y_{1: K}\right) \\
& \quad \propto p\left(\alpha_{1}, \beta_{1}, \alpha_{2}, \beta_{2}, \gamma_{1}, \gamma_{2}, \gamma_{3}, e_{1}, \rho, P_{0: K}, \phi_{0: K}, \sigma_{0: K}\right) \\
& \quad \cdot p\left(Y_{1: K} \mid \alpha_{1}, \beta_{1}, \alpha_{2}, \beta_{2}, \gamma_{1}, \gamma_{2}, \gamma_{3}, e_{1}, \rho, P_{1: K}, \phi_{1: K}, \sigma_{1: K}\right),
\end{aligned}
$$

where the joint prior density is

$$
\begin{aligned}
p( & \left.\alpha_{1}, \beta_{1}, \alpha_{2}, \beta_{2}, \gamma_{1}, \gamma_{2}, \gamma_{3}, e_{1}, \rho, P_{0: K}, \phi_{0: K}, \sigma_{0: K}\right) \\
= & p\left(\alpha_{1}, \beta_{1}, \alpha_{2}, \beta_{2}, \gamma_{1}, \gamma_{2}, \gamma_{3}, e_{1}, \rho\right) p\left(P_{0}\right) \\
& \cdot \prod_{k=1}^{K} p\left(P_{k} \mid P_{k-1}, \alpha_{1}, \beta_{1}, \alpha_{2}, \beta_{2}, \gamma_{1}, \gamma_{2}, \gamma_{3}, e_{1}, \rho\right) \\
& \cdot p\left(\phi_{0}\right) \\
& \cdot \prod_{k=1}^{K} p\left(\phi_{k} \mid \phi_{k-1}, \alpha_{1}, \beta_{1}, \alpha_{2}, \beta_{2}, \gamma_{1}, \gamma_{2}, \gamma_{3}, e_{1}, \rho\right) \\
& \cdot p\left(\sigma_{0}\right) \\
& \cdot \prod_{k=1}^{K} p\left(\sigma_{k} \mid \sigma_{k-1}, \alpha_{1}, \beta_{1}, \alpha_{2}, \beta_{2}, \gamma_{1}, \gamma_{2}, \gamma_{3}, e_{1}, \rho\right)
\end{aligned}
$$

and the likelihood function is

$$
\begin{aligned}
p & \left(Y_{1: K} \mid \alpha_{1}, \beta_{1}, \alpha_{2}, \beta_{2}, \gamma_{1}, \gamma_{2}, \gamma_{3}, e_{1}, \rho, P_{1: K}, \phi_{1: K}, \sigma_{1: K}\right) \\
& =\prod_{k=1}^{K} p\left(Y_{k} \mid P_{k}, e_{1}\right) .
\end{aligned}
$$

A Markov chain is generated by Gibbs sampling and its stationary distribution is the joint posterior distribution (21). Under a given prior probability density $p(\boldsymbol{\theta})$ for $\boldsymbol{\theta}$, it is now possible to efficiently sample the posterior density $p(P, \phi, \sigma, \boldsymbol{\theta} \mid Y)$ by the MCMC technique. We develop the following sampling algorithm for the model (13).

Step 1. Initialize

$$
\alpha_{1}^{(0)}, \beta_{1}^{(0)}, \alpha_{2}^{(0)}, \beta_{2}^{(0)}, \gamma_{1}^{(0)}, \gamma_{2}^{(0)}, \gamma_{3}^{(0)}, e_{1}^{(0)}, \rho^{(0)}, P_{0: K}^{0}, \phi_{0: K}^{0}, \sigma_{0: K}^{0} .
$$

Step 2. Sample $\alpha_{1}^{(1)}$ from

$$
\begin{aligned}
& p\left(\alpha_{1} \mid \beta_{1}^{(0)}, \alpha_{2}^{(0)}, \beta_{2}^{(0)}, \gamma_{1}^{(0)}, \gamma_{2}^{(0)}, \gamma_{3}^{(0)}, e_{1}^{(0)}, \rho^{(0)}, P_{0: K}^{0}, \phi_{0: K}^{0},\right. \\
& \left.\sigma_{0: K}^{0}, Y_{1: K}\right) .
\end{aligned}
$$

Step 3. Sample $\beta_{1}^{(1)}$ from

$$
\begin{aligned}
& p\left(\beta_{1} \mid \alpha_{1}^{(1)}, \alpha_{2}^{(0)}, \beta_{2}^{(0)}, \gamma_{1}^{(0)}, \gamma_{2}^{(0)}, \gamma_{3}^{(0)}, e_{1}^{(0)}, \rho^{(0)}, P_{0: K}^{0}, \phi_{0: K}^{0},\right. \\
& \left.\sigma_{0: K}^{0}, Y_{1: K}\right) .
\end{aligned}
$$

Step 4. Sample $\alpha_{2}^{(1)}$ from

$$
\begin{aligned}
& p\left(\alpha_{2} \mid \alpha_{1}^{(1)}, \beta_{1}^{(1)}, \beta_{2}^{(0)}, \gamma_{1}^{(0)}, \gamma_{2}^{(0)}, \gamma_{3}^{(0)}, e_{1}^{(0)}, \rho^{(0)}, P_{0: K}^{0}, \phi_{0: K}^{0},\right. \\
& \left.\sigma_{0: K}^{0}, Y_{1: K}\right) .
\end{aligned}
$$

Step 5. Sample $\beta_{2}^{(1)}$ from

$$
\begin{aligned}
& p\left(\beta_{2} \mid \alpha_{1}^{(1)}, \alpha_{2}^{(1)}, \beta_{1}^{(1)}, \gamma_{1}^{(0)}, \gamma_{2}^{(0)}, \gamma_{3}^{(0)}, e_{1}^{(0)}, \rho^{(0)}, P_{0: K}^{0}, \phi_{0: K}^{0},\right. \\
& \left.\quad \sigma_{0: K}^{0}, Y_{1: K}\right) .
\end{aligned}
$$

Step 6. Sample $\gamma_{1}^{(1)}$ from

$$
\begin{aligned}
& p\left(\gamma_{1} \mid \alpha_{1}^{(1)}, \alpha_{2}^{(1)}, \beta_{1}^{(1)}, \beta_{2}^{(1)}, \gamma_{2}^{(0)}, \gamma_{3}^{(0)}, e_{1}^{(0)}, \rho^{(0)}, P_{0: K}^{0}, \phi_{0: K}^{0},\right. \\
& \left.\sigma_{0: K}^{0}, Y_{1: K}\right) .
\end{aligned}
$$

Step 7. Sample $\gamma_{2}^{(1)}$ from

$$
\begin{aligned}
& p\left(\gamma_{2} \mid \alpha_{1}^{(1)}, \alpha_{2}^{(1)}, \beta_{1}^{(1)}, \beta_{2}^{(1)}, \gamma_{1}^{(1)}, \gamma_{3}^{(0)}, e_{1}^{(0)}, \rho^{(0)}, P_{0: K}^{0}, \phi_{0: K}^{0},\right. \\
& \left.\quad \sigma_{0: K}^{0}, Y_{1: K}\right) .
\end{aligned}
$$

Step 8. Sample $\gamma_{3}^{(1)}$ from

$$
\begin{aligned}
& p\left(\gamma_{3} \mid \alpha_{1}^{(1)}, \alpha_{2}^{(1)}, \beta_{1}^{(1)}, \beta_{2}^{(1)}, \gamma_{1}^{(1)}, \gamma_{2}^{(1)}, e_{1}^{(0)}, \rho^{(0)}, P_{0: K}^{0}, \phi_{0: K}^{0},\right. \\
& \left.\sigma_{0: K}^{0}, Y_{1: K}\right) .
\end{aligned}
$$

Step 9. Sample $e_{1}^{(1)}$ from

$$
\begin{aligned}
& p\left(e_{1} \mid \alpha_{1}^{(1)}, \alpha_{2}^{(1)}, \beta_{1}^{(1)}, \beta_{2}^{(1)}, \gamma_{1}^{(1)}, \gamma_{2}^{(1)}, \gamma_{3}^{(1)}, \rho^{(0)}, P_{0: K}^{0}, \phi_{0: K}^{0}, \sigma_{0: K}^{0},\right. \\
& \left.\quad Y_{1: K}\right) .
\end{aligned}
$$

Step 10. Sample $\rho^{(1)}$ from

$$
\begin{aligned}
& p\left(\rho \mid \alpha_{1}^{(1)}, \alpha_{2}^{(1)}, \beta_{1}^{(1)}, \beta_{2}^{(1)}, \gamma_{1}^{(1)}, \gamma_{2}^{(1)}, \gamma_{3}^{(1)}, e_{1}^{(1)}, P_{0: K}^{0}, \phi_{0: K}^{0}, \sigma_{0: K}^{0},\right. \\
& \left.\quad Y_{1: K}\right) .
\end{aligned}
$$

Step 11. For $k=0,1,2, \ldots, K$, sample $P_{k}^{(1)}$ from

$$
\begin{aligned}
p( & P_{k} \mid \alpha_{1}^{(1)}, \alpha_{2}^{(1)}, \beta_{1}^{(1)}, \beta_{2}^{(1)}, \gamma_{1}^{(1)}, \gamma_{2}^{(1)}, \gamma_{3}^{(1)}, e_{1}^{(1)}, \rho^{(1)}, P_{-k}, \phi_{0: K}^{0}, \\
& \left.\sigma_{0: K}^{0}, Y_{1: K}\right),
\end{aligned}
$$

where $P_{-k}=\left(P_{1}^{(1)}, P_{2}^{(1)}, \ldots, P_{k-1}^{(1)}, P_{k+1}^{(0)}, \ldots, P_{K}^{(0)}\right)$.

Step 12. For $k=0,1,2, \ldots, K$, sample $\phi_{k}^{(1)}$ from

$$
\begin{aligned}
& p\left(\phi_{k} \mid \alpha_{1}^{(1)}, \alpha_{2}^{(1)}, \beta_{1}^{(1)}, \beta_{2}^{(1)}, \gamma_{1}^{(1)}, \gamma_{2}^{(1)}, \gamma_{3}^{(1)}, e_{1}^{(1)}, \rho^{(1)}, P_{0: K}^{(1)}, \phi_{-k},\right. \\
& \left.\sigma_{0: K}^{0}, Y_{1: K}\right),
\end{aligned}
$$

where $\phi_{-k}=\left(\phi_{1}^{(1)}, \phi_{2}^{(1)}, \ldots, \phi_{k-1}^{(1)}, \phi_{k+1}^{(0)}, \ldots, \phi_{K}^{(0)}\right)$.

Step 13. For $k=0,1,2, \ldots, K$, sample $\sigma_{k}^{(1)}$ from

$$
\begin{aligned}
& p\left(\sigma_{k} \mid \alpha_{1}^{(1)}, \alpha_{2}^{(1)}, \beta_{1}^{(1)}, \beta_{2}^{(1)}, \gamma_{1}^{(1)}, \gamma_{2}^{(1)}, \gamma_{3}^{(1)}, e_{1}^{(1)}, \rho^{(1)}, P_{0: K}^{(1)}, \phi_{0: K}^{(1)},\right. \\
& \left.\sigma_{-k}, Y_{1: K}\right),
\end{aligned}
$$

where $\sigma_{-k}=\left(\sigma_{1}^{(1)}, \sigma_{2}^{(1)}, \ldots, \sigma_{k-1}^{(1)}, \sigma_{k+1}^{(0)}, \ldots, \sigma_{K}^{(0)}\right)$.

Step 14. Repeat the above procedure and stop until the number of iterations is $M$, which must be large enough to guarantee that the Markov chain reaches stationary distribution. 


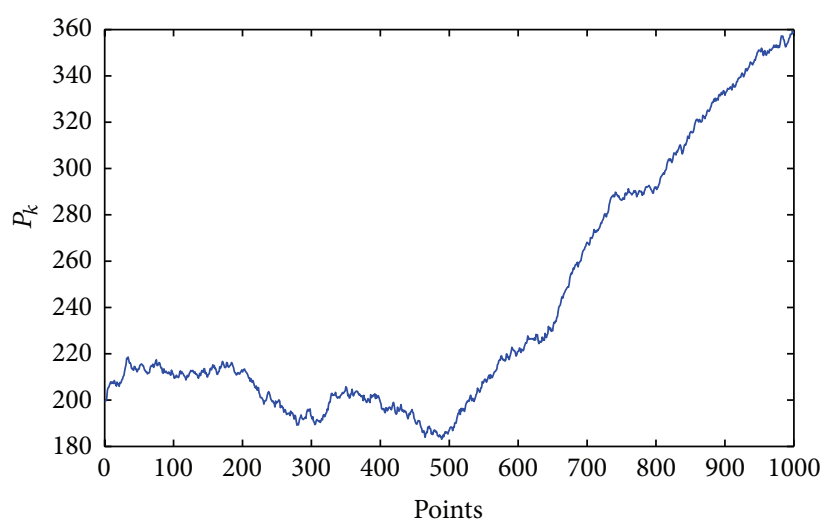

FIgURE 1: Simulation price data $P_{k}$ made from model (13).

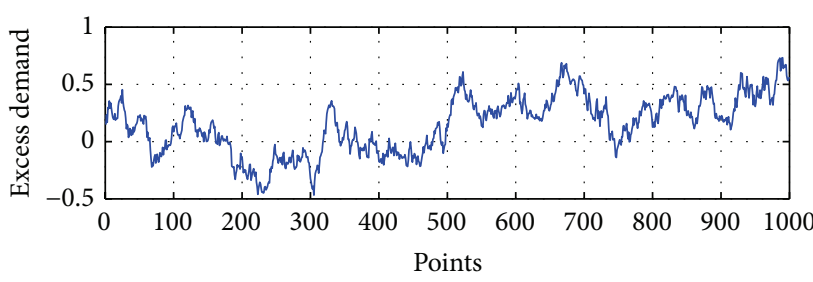

(a)

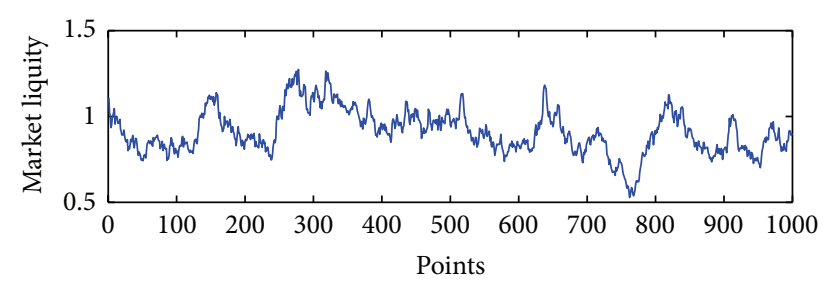

(b)

Figure 2: Simulation data $\phi_{k}, e^{\sigma_{k}}$ made from model (13).

\section{Simulation Study}

For simulation study of the proposed leverage market microstructure model by the MCMC algorithm, 1000 observations from model (13) are generated with the unknown parameters $\alpha_{1}=0.0013, \alpha_{2}=-0.0035,1+\beta_{1}=0.98$, $1+\beta_{2}=0.97, \gamma_{1}=0.047, \gamma_{2}=0.032$, and $\rho=-0.2$ and the known parameters $\gamma_{3}=1, e_{1}=1 e-5$. The initial value $P_{0}$, $\phi_{0}$, and $\sigma_{0}$ are taken as $199.3,0.1489$, and 0.1024 , respectively. Figures 1 and 2 show the simulation data made from model (13).

Meyer and $\mathrm{Yu}$ [35] found that the statistical inference is insensitive to changing in the prior distributions of the parameters. Hence, the similar prior distributions are assumed as follows:

$$
\begin{aligned}
\alpha_{1} & \sim N\left(0,10^{-2}\right) \\
1+\beta_{1} & \sim \operatorname{Beta}(20,1.5) \\
\alpha_{2} & \sim N\left(0,10^{-2}\right) \\
1+\beta_{2} & \sim \operatorname{Beta}(20,1.5) \\
\gamma_{1}^{-2} & \sim \operatorname{Gamma}(2.5,0.0055), \\
\gamma_{2}^{-2} & \sim \operatorname{Gamma}(2.5,0.00256), \\
\rho & \sim U(-1,1) .
\end{aligned}
$$

The $\operatorname{Beta}(20,1.5)$ prior distribution for $1+\beta_{1}, 1+\beta_{2}$ implies that the mean and standard deviation are $0.93,0.0029$.
The means and standard deviation of $\operatorname{Gamma}(2.5,0.0055)$ and $\operatorname{Gamma}(2.5,0.00256)$ are $454.55,287.48$ and 976.56, 617.63 , respectively.

In the experiment, a Markov chain is run for 20000 iterations and the initial 6000 iterations are discarded as the burn-in period to ensure convergence. Figure 3 shows the sample paths and the posterior densities for each parameter. After discarding samples in the burn-in period, the sample paths look stable, indicating that our sampling method efficiency produces uncorrelated samples. Table 1 gives the estimates for posterior means, standard deviations, and the 95\% credible intervals. All estimated posterior means are close to the true values and the true values are contained in the $95 \%$ credible intervals. The simulation results show that Bayes estimates have very good sampling properties.

\section{Empirical Study}

5.1. Stock Index in China. In this subsection, two stock indices in China are employed to fit the leverage market microstructure model, namely, the Shanghai composite index and Shenzhen component index. The sample period is from January 2, 1997, to June 30, 2004. The sample size is 1801 for each series. The observation data $P_{k}$ is computed as $P_{k}=$ $100 \times \log Z_{k}$, where $Z_{k}$ denotes the (closing) spot price. Figure 4 shows the observations. Table 2 summarizes the descriptive statistics of the two indices. These descriptive statistics include sample means, standard deviations, skewness, kurtosis, and the Jarque-Bera statistics. 

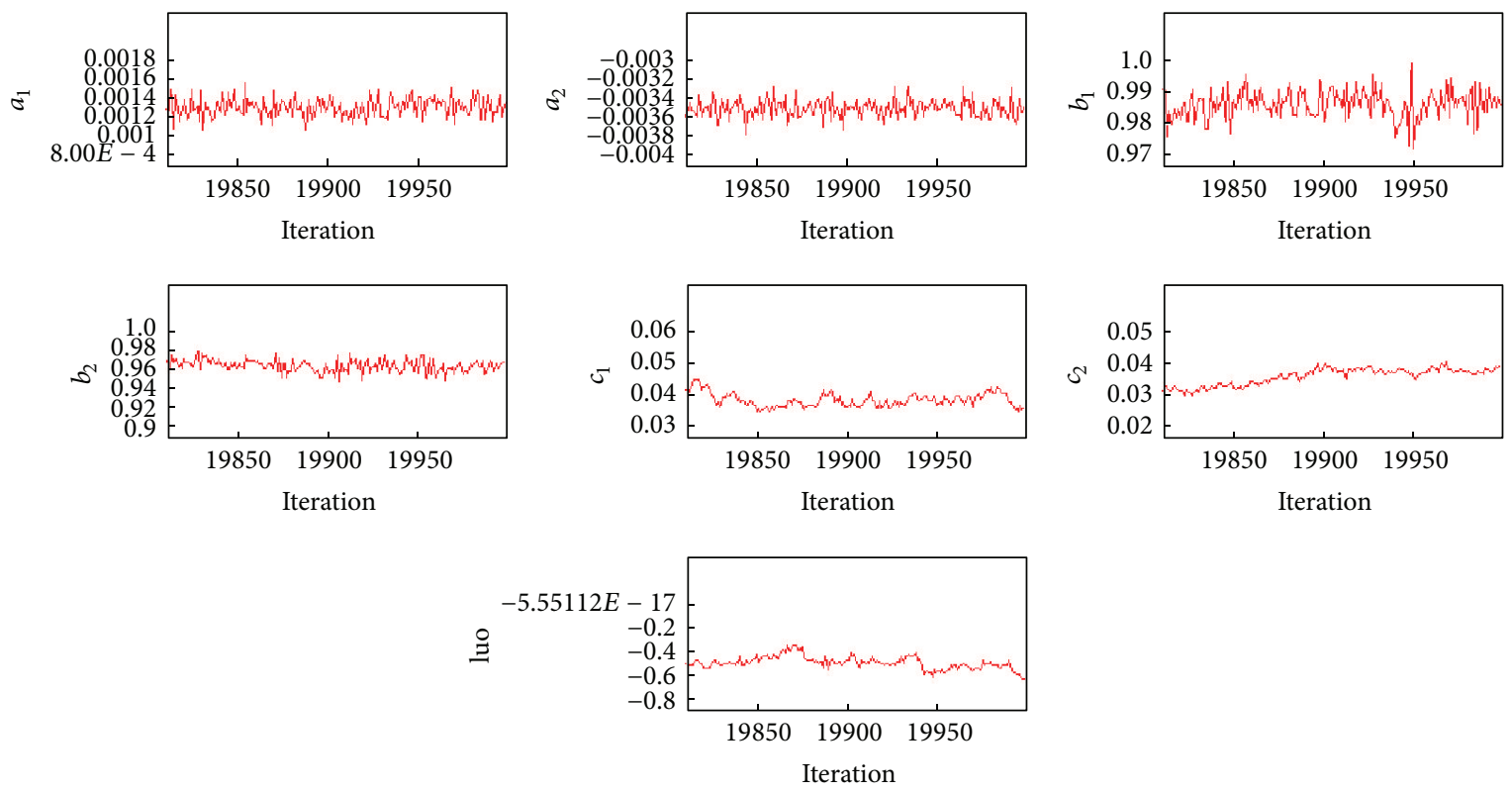

(a) Sample paths
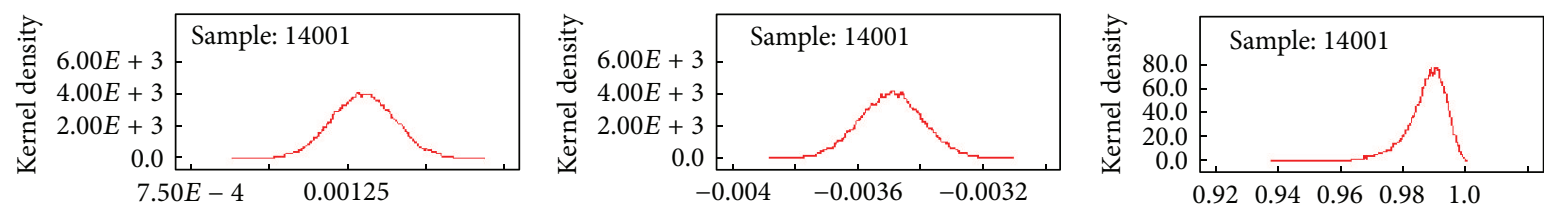

$a_{1}$

$a_{2}$

$b_{1}$
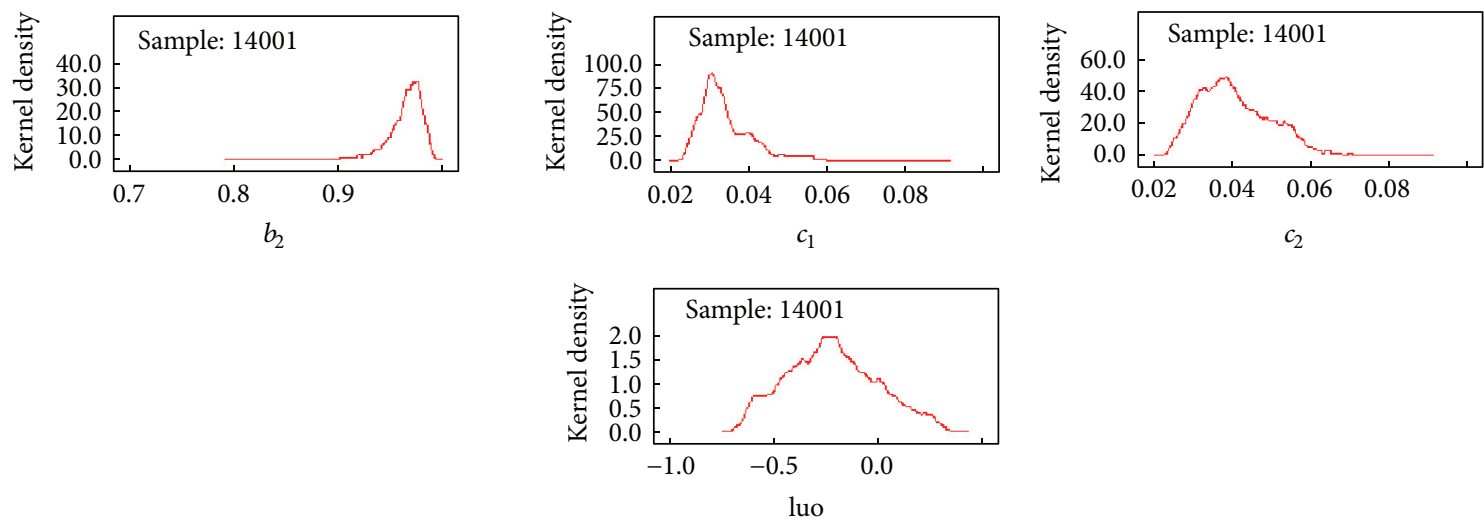

(b) Posterior densities

Figure 3: Path and density estimates of the leverage market microstructure model for simulated data (where $a_{1}=\alpha_{1}, a_{2}=\alpha_{2}, b_{1}=1+\beta_{1}$, $b_{2}=1+\beta_{2}, c_{1}=\gamma_{1}, c_{2}=\gamma_{2}$, and luo $=\rho$ ).

TABLE 1: Estimation results for simulated data

\begin{tabular}{lcccccc}
\hline Parameter & True & Mean & SD & 2.5 percentile & Median & 97.5 percentile \\
\hline$\alpha_{1}$ & 0.0013 & 0.001304 & $1.002 E-4$ & 0.001111 & 0.001304 & 0.001505 \\
$\alpha_{2}$ & -0.0035 & -0.003494 & $9.956 E-5$ & -0.003688 & -0.00349 & -0.003296 \\
$1+\beta_{1}$ & 0.98 & 0.9874 & 0.006541 & 0.971 & 0.9866 & 0.9968 \\
$1+\beta_{2}$ & 0.97 & 0.9628 & 0.02023 & 0.906 & 0.9672 & 0.9861 \\
$\gamma_{1}$ & 0.047 & 0.04103 & 0.009441 & 0.02662 & 0.03948 & 0.06083 \\
$\gamma_{2}$ & 0.032 & 0.03541 & 0.008835 & 0.02542 & 0.03296 & 0.059 \\
$\rho$ & -0.2 & -0.2182 & 0.0216 & -0.6038 & -0.2274 & 0.2266 \\
\hline
\end{tabular}




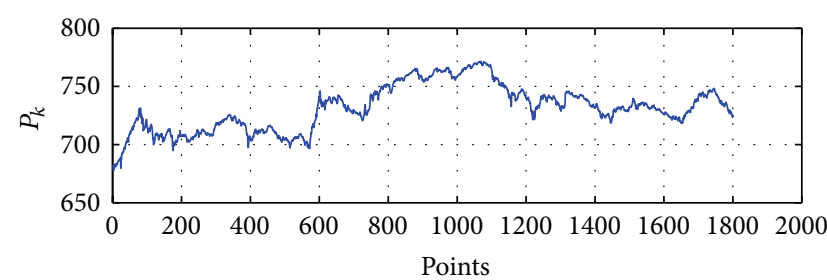

(a)

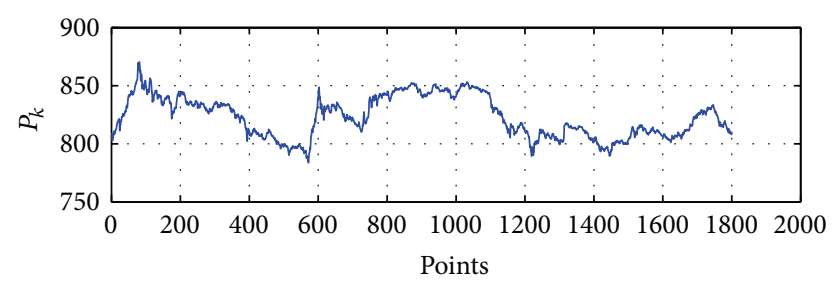

(b)

Figure 4: The observation data ((a): Shanghai composite index, (b): Shenzhen component index).

TABLE 2: Descriptive statistics for Shanghai composite index and Shenzhen component index.

\begin{tabular}{lccccc}
\hline Index & Mean & Standard deviation & Skewness & Kurtosis & Jarque-Bera statistics \\
\hline Shanghai composite index & 0.0233 & 1.552 & -0.0835 & 9.172 & 2859.554 \\
Shenzhen component index & -0.0000453 & 1.705 & 0.00312 & 8.362 & 2156.039 \\
\hline
\end{tabular}

Table 2 shows that the standard deviation is 1.552 for the Shanghai composite index and 1.705 for the Shenzhen component index, which indicates that the volatility level of the latter is higher than that of the former. The skewness of the Shanghai composite index is negative, while the Shenzhen component index has slightly positive skewness. The kurtosis of each index is clearly larger than that of a normal distribution. Based on Jarque-Bera statistics, the two indices strongly reject normality hypothesis. So, the statistics results show the skewness and heavy tailedness of empirical returns distribution of the data.

We consider model (13) to be fitted to the data. The prior distributions for the parameters are the same as the simulation study in Section 4. The parameter estimates for the leverage market microstructure model based on 100000 iterations after a burn-in of 20000 are given in Table 3. Figure 5 shows the sample paths and the posterior densities for each parameter.

It is shown in Table 3 that the posterior means of $1+$ $\beta_{2}$ are close to one, which indicates the well-known high persistence of volatility in stock markets. The posterior means of $\rho$ are -0.3475 and -0.2586 , respectively. The $95 \%$ posterior credibility intervals for $\rho$ are $[-0.4788,-0.2154]$ and $[-0.4192,-0.108]$ and do not contain zero, which indicates the presence of the credible leverage effect in Chinese stock market. Compared with the Shenzhen component index, the Shanghai composite index has more significant leverage effect, which is consistent with the previous statistical results.

According to the estimated parameters, the volatility level of the two indices can be obtained, respectively, as follows:

$$
\begin{aligned}
& \mu_{1}=-\frac{-5.763 \times 10^{-4}}{0.9251-1}=-7.694 \times 10^{-3}, \\
& \mu_{2}=-\frac{-0.002349}{0.9493-1}=-4.633 \times 10^{-2} .
\end{aligned}
$$

Obviously, the volatility level of the Shanghai composite index $\left(-7.694 \times 10^{-3}\right)$ is lower than that of the Shenzhen component index $\left(-4.633 \times 10^{-2}\right)$, which is also coordinated with the previous statistical results. This is mainly because the Shenzhen stock is small-cap stocks, while the Shanghai stock is large stocks.

5.2. Stock Index in U.S. In this subsection, we analyze the series which contains 2519 observations of the Standard and Poor's 500 Composite (S\&P500) index from January 2, 2002, to December 30, 2011. Figure 6 shows the observations, and Figure 7 gives the probability histogram of stock index returns. Table 4 lists descriptive statistics of the index. From Table 4, we can see that the series possesses the characteristic of excess kurtosis, skewness, and volatility asymmetry, which prompts us to analyze the data under the assumption of skewness and volatility asymmetry.

Before we proceed to the estimation of the leverage market microstructure model on the S\&P500 index, the prior distributions for the parameters are the same as the simulation study in Section 5.1. To guarantee the convergence of the parameters, the MCMC algorithm is running for 110000 iterations, and the initial 60000 samples are discarded. Figure 8 shows the sample paths and posterior densities for each parameter. Table 5 gives the estimated posterior means, the standard deviations, and the $95 \%$ credible intervals.

From the estimation results of Table 5, it is seen that the standard deviation and MC error are small and MC error is 1-2 order of magnitude smaller than the standard deviation, which indicates that the MCMC estimation is accurate. The estimate of $1+\beta_{2}$ shows the high persistence of volatilities varying from 0.9795 to 0.9908 , and the negative value of $\rho$ (ranging from -0.8650 to -0.6874 ) implies the credible existence of leverage effects for the S\&P500.

Compared with the estimates of Chinese stock market in the previous section, American stock market has stronger leverage effect. This is mainly because there is $\pm 10 \%$ increase in the limit for Chinese stock market; even, on the extreme condition, the largest decline in daily stock price is only $10 \%$. 

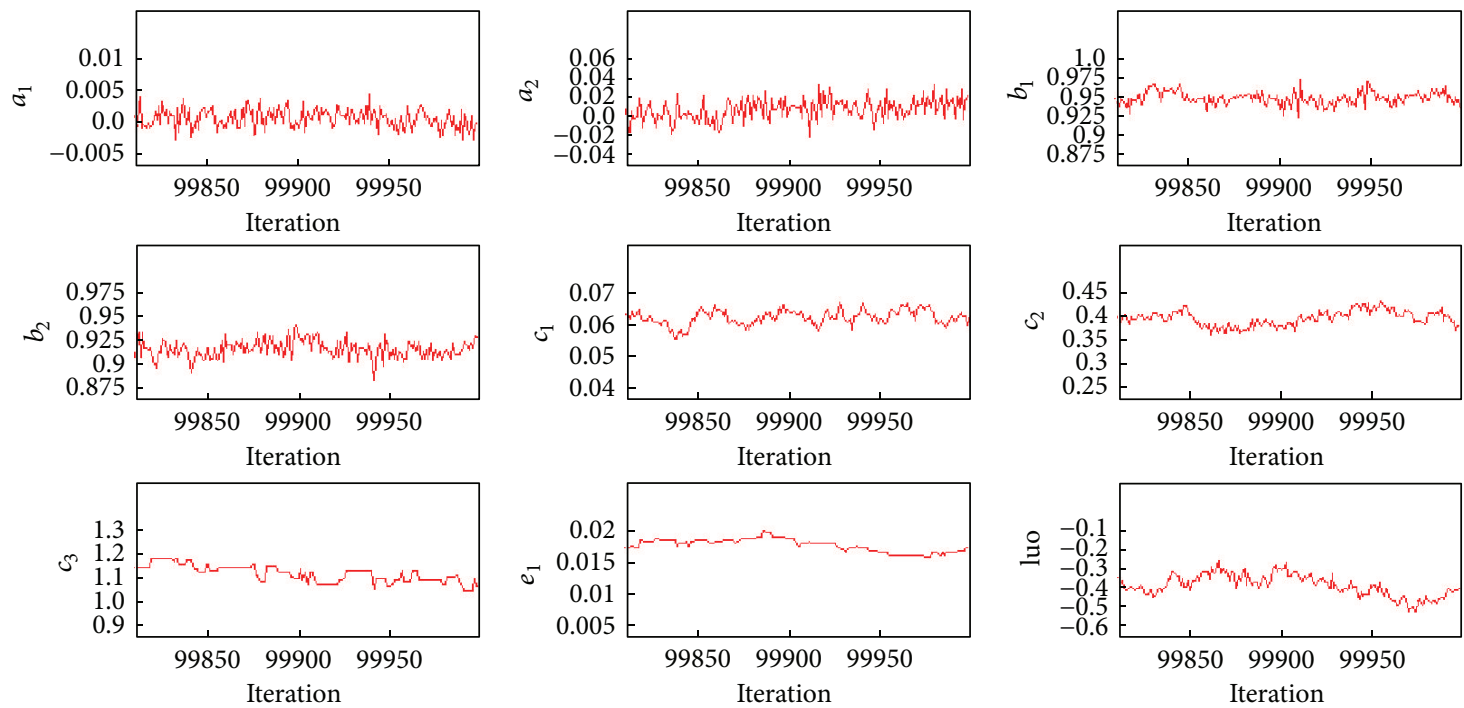

(a) Sample paths for Shanghai composite index
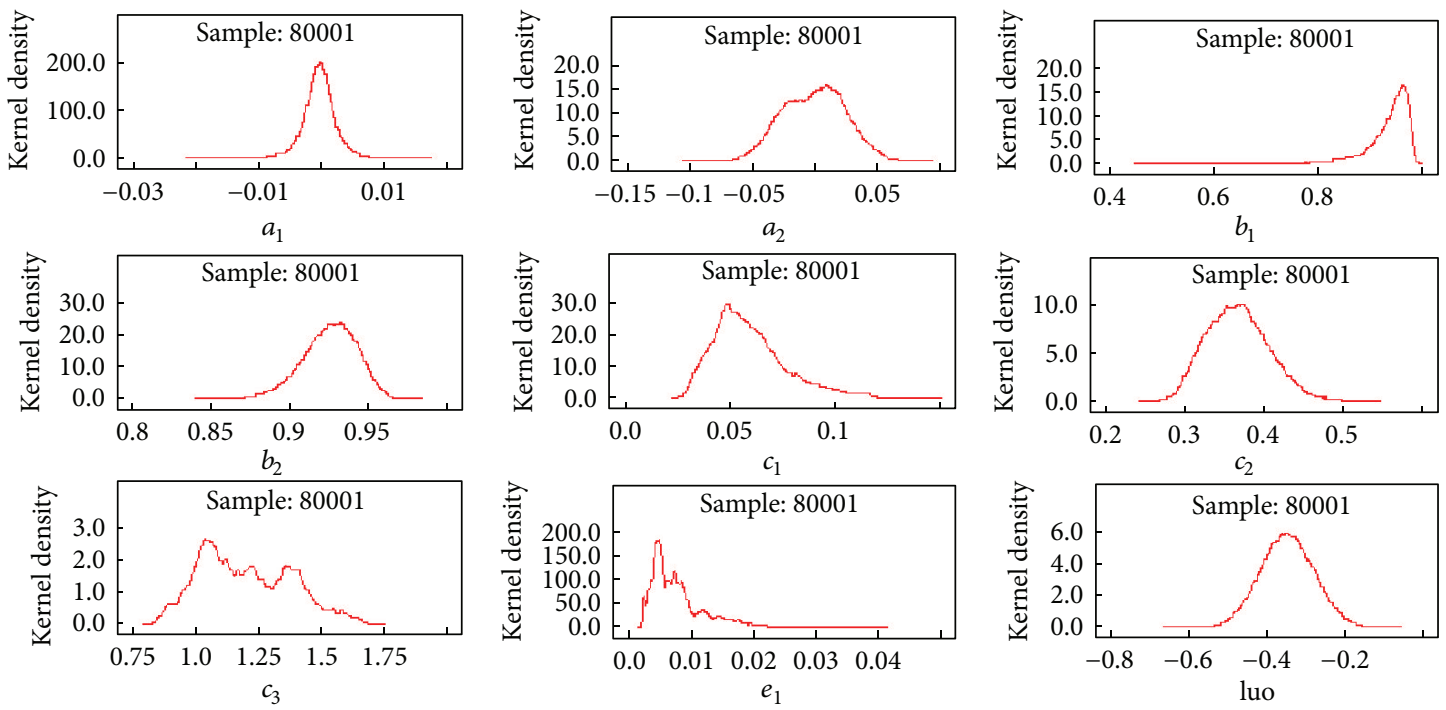

(b) Sample posterior densities for Shanghai composite index
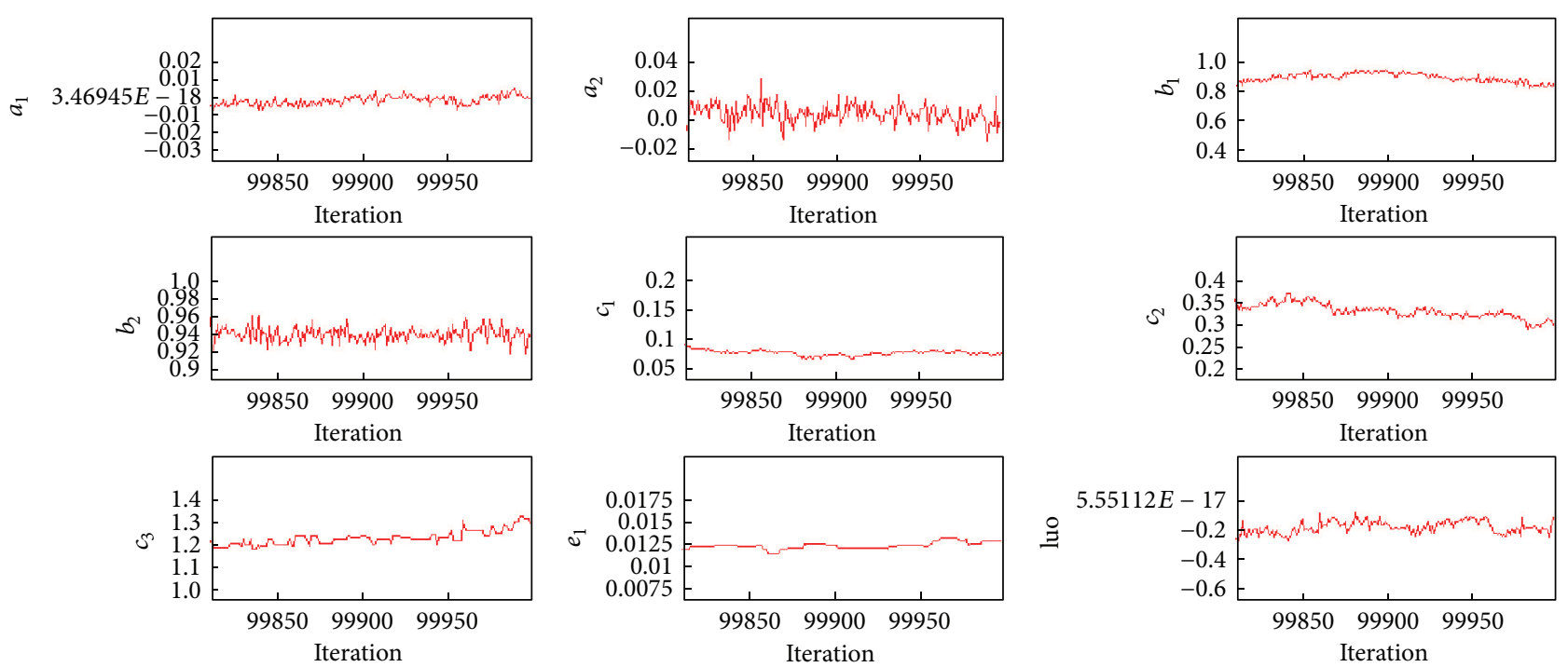

(c) Sample paths for Shenzhen component index

Figure 5: Continued. 

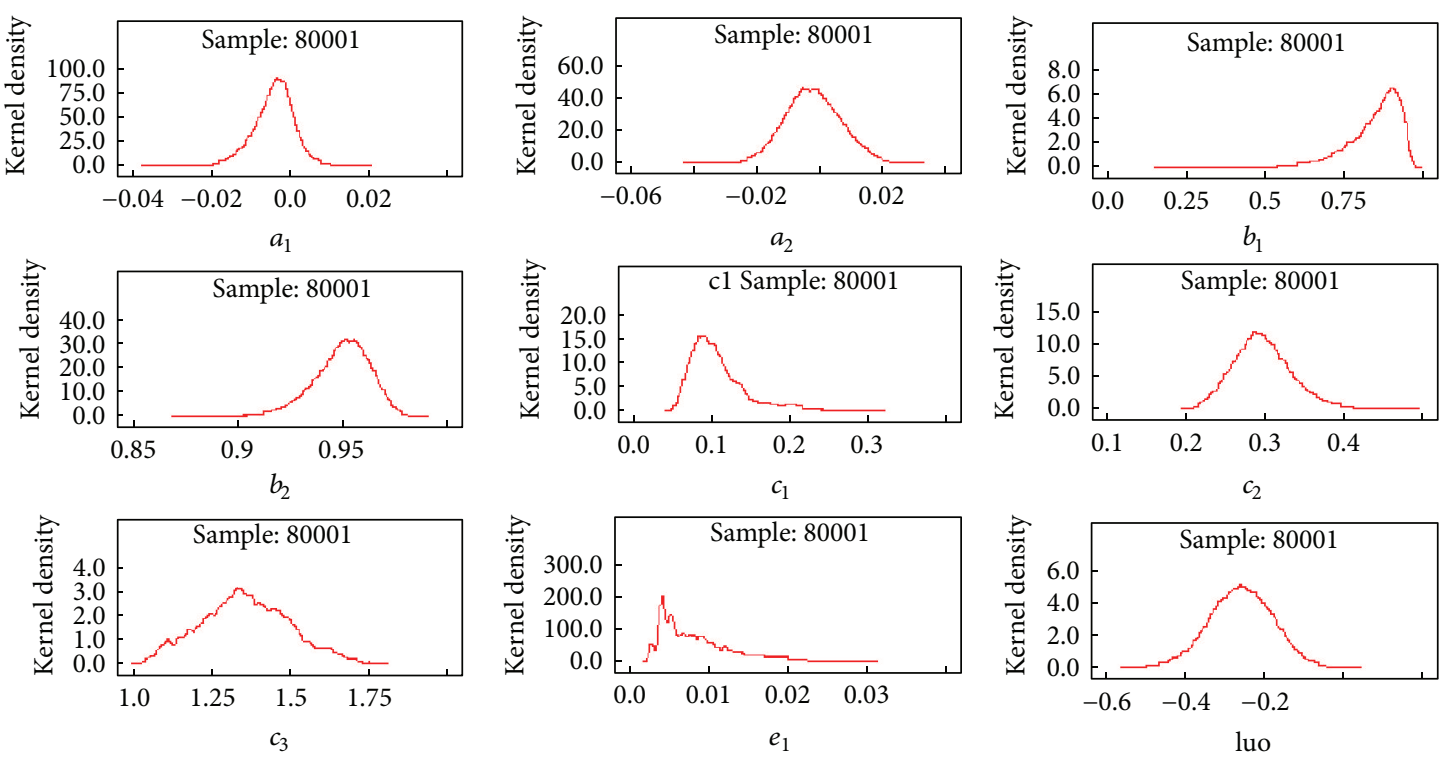

(d) Sample posterior densities for Shenzhen component index

FIGURE 5: Sample path and density estimates for two indices.

TABLE 3: Empirical estimation results for two indices.

\begin{tabular}{|c|c|c|c|c|c|c|c|}
\hline Index & Parameter & Mean & $\mathrm{SD}$ & MC error & 2.5 percentile & Median & 97.5 percentile \\
\hline \multirow{9}{*}{$\begin{array}{l}\text { Shanghai } \\
\text { composite } \\
\text { index }\end{array}$} & $\alpha_{1}$ & $-3.588 E-4$ & 0.002697 & $1.124 E-4$ & -0.00591 & $-3.112 E-4$ & 0.004956 \\
\hline & $\alpha_{2}$ & $-5.763 E-4$ & 0.02449 & 0.001346 & -0.04714 & $4.838 E-4$ & 0.04552 \\
\hline & $1+\beta_{1}$ & 0.9304 & 0.04995 & 0.002694 & 0.7924 & 0.9443 & 0.9787 \\
\hline & $1+\beta_{2}$ & 0.9251 & 0.01689 & $7.082 E-4$ & 0.8887 & 0.9264 & 0.9543 \\
\hline & $\gamma_{1}$ & 0.05965 & 0.01763 & $9.719 E-4$ & 0.03364 & 0.05658 & 0.1036 \\
\hline & $\gamma_{2}$ & 0.3679 & 0.03929 & 0.001917 & 0.2996 & 0.3663 & 0.4496 \\
\hline & $\gamma_{3}$ & 1.199 & 0.18 & 0.01056 & 0.9034 & 1.177 & 1.57 \\
\hline & $e_{1}$ & 0.008126 & 0.004677 & $2.736 E-4$ & 0.002771 & 0.006927 & 0.02006 \\
\hline & $\rho$ & -0.3475 & 0.06774 & 0.002665 & -0.4788 & -0.3478 & -0.2154 \\
\hline \multirow{9}{*}{$\begin{array}{l}\text { Shenzhen } \\
\text { component } \\
\text { index }\end{array}$} & $\alpha_{1}$ & -0.004039 & 0.005084 & $1.77 E-4$ & -0.01513 & -0.003641 & 0.005447 \\
\hline & $\alpha_{2}$ & -0.002349 & 0.008672 & $3.799 E-4$ & -0.0188 & -0.002569 & 0.01492 \\
\hline & $1+\beta_{1}$ & 0.8445 & 0.08805 & 0.004545 & 0.6134 & 0.8668 & 0.951 \\
\hline & $1+\beta_{2}$ & 0.9493 & 0.01366 & $6.03 E-4$ & 0.9185 & 0.9508 & 0.9721 \\
\hline & $\gamma_{1}$ & 0.1083 & 0.03612 & 0.001988 & 0.06241 & 0.1003 & 0.2082 \\
\hline & $\gamma_{2}$ & 0.2973 & 0.0369 & 0.001955 & 0.2318 & 0.2947 & 0.3778 \\
\hline & $\gamma_{3}$ & 1.36 & 0.1379 & 0.007978 & 1.099 & 1.356 & 1.639 \\
\hline & $e_{1}$ & 0.008615 & 0.004806 & $2.822 E-4$ & 0.002938 & 0.007354 & 0.02097 \\
\hline & $\rho$ & -0.2586 & 0.0791 & 0.003338 & -0.4192 & -0.2576 & -0.108 \\
\hline
\end{tabular}

However, the stock market in the United States has no such restrictions, and the fall in the extreme case far exceeds $10 \%$.

\section{Model Comparison}

In order to compare the competing leverage SV model and the leverage market microstructure model in terms of the Deviance Information Criterion (DIC), the above three sets of stock indices were fitted by the leverage stochastic volatility model. The leverage stochastic volatility (SV) model can be formulated as follows $[6,35]$ :

$$
\begin{aligned}
& y_{t}=e^{h_{t} / 2} \xi_{t}, \\
& h_{t}=\mu+\varphi\left(h_{t-1}-\mu\right)+\tau \eta_{t},
\end{aligned}
$$

where $y_{t}$ is the asset return, $h_{t}$ is the unobserved logvolatility, $\mu$ represents the intercept, $|\varphi|<1$ is the persistence 
TABLE 4: Descriptive statistics for S\&P500.

\begin{tabular}{lccccc}
\hline Index & Mean & Standard deviation & Skewness & Kurtosis & Jarque-Bera statistics \\
\hline S\&P500 & 0.003391 & 1.3883 & -0.1832 & 11.1175 & 6927.3851 \\
\hline
\end{tabular}

TABLE 5: Estimation results for S\&P500.

\begin{tabular}{lcccccc}
\hline Parameter & Mean & SD & MC error & 2.5 percentile & Median & 97.5 percentile \\
\hline$\alpha_{1}$ & 0.004191 & 0.006326 & $3.599 E-4$ & -0.007307 & 0.003335 & 0.01872 \\
$\alpha_{2}$ & 0.00771 & 0.003923 & $1.984 E-4$ & $1.37 E-4$ & 0.007663 & 0.01572 \\
$1+\beta_{1}$ & 0.6338 & 0.1335 & 0.00753 & 0.3478 & 0.6494 & 0.9858 \\
$1+\beta_{2}$ & 0.9857 & 0.002876 & $1.337 E-4$ & 0.9795 & 0.0401 \\
$\gamma_{1}$ & 0.04162 & 0.01056 & $6.432 E-4$ & 0.0253 & 0.1569 & 0.06742 \\
$\gamma_{2}$ & 0.1584 & 0.01493 & $9.303 E-4$ & 0.1311 & 0.7633 & 0.1892 \\
$\gamma_{3}$ & 0.7655 & 0.05411 & 0.003546 & 0.6723 & 0.006704 & 0.01882 \\
$e_{1}$ & 0.0082 & 0.004425 & $2.93 E-4$ & 0.003277 & -0.7833 \\
$\rho$ & -0.7805 & 0.04531 & 0.002657 & -0.8650 & -0.6874 \\
\hline
\end{tabular}

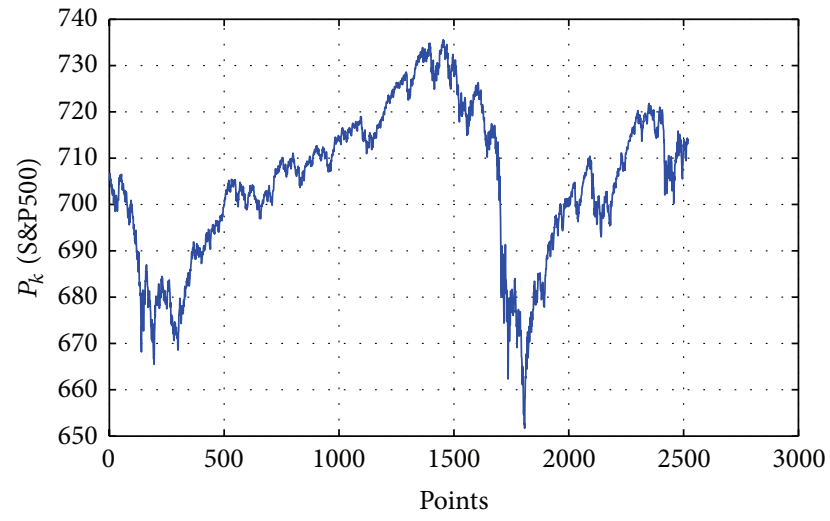

FIgURE 6: S\&P500 time series $P_{k}$.

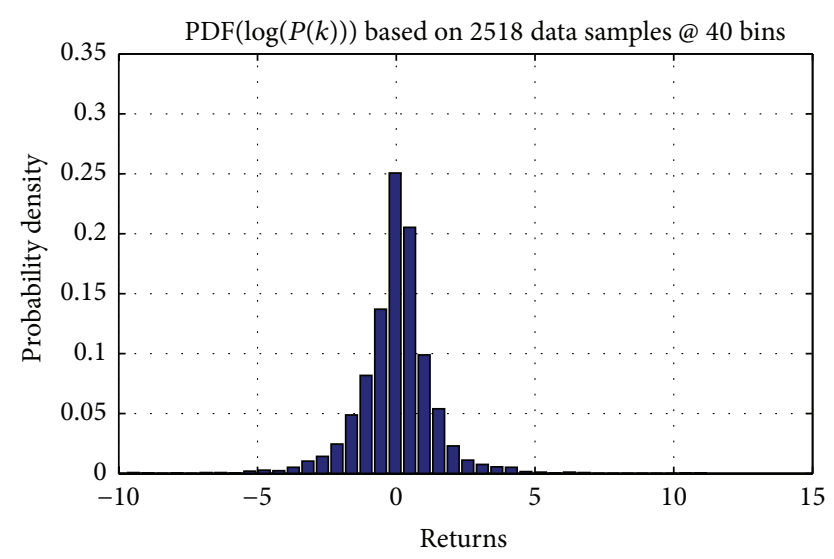

FIGURE 7: The probability histogram of S\&P500.

in the volatility, $\tau$ is the log-volatility conditional standard deviation, and $\xi_{t}, \eta_{t}$ are iid $N(0,1)$ and $\operatorname{corr}\left(\xi_{t}, \eta_{t+1}\right)=\rho$. The leverage effect measured by the correlation coefficient $\rho$ is expected to be negative.
For the leverage SV model, the prior distributions for $\left(\mu, \varphi, \tau^{2}, \rho\right)$ are set as $\mu \sim N(0,10),(\varphi+1) / 2 \sim \operatorname{Beta}(20,1.5)$, $1 / \tau^{2} \sim \operatorname{Gamma}(2.5,0.025)$, and $\rho \sim U(-1,1)$, which are the same as Meyer and $\mathrm{Yu}$ [35]. The numbers of MCMC iterations for the three indices are the same as Section 5. Table 6 reports the parameter estimates of the leverage SV model for the three indices, and Table 7 lists DIC together with $\bar{D}$ and $P_{D}$ for the two models.

First, the $\rho$ values of the three indices evaluated from the leverage SV model are significantly negative and close to the estimation of the leverage market microstructure model, which is in agreement with the fact that there exists the leverage effect in stock return data. Also, the leverage effect is more significant in the United States stock market $(\rho=$ -0.5942) than that in Chinese stock market ( $\rho=-0.3053$ and -0.2532 ), which is consistent with the previous statistical results. Second, for both two models, the posterior means $\varphi$ and $1+\beta_{2}$ are close to one, which implies a well-known high persistence of volatility on stock returns. Third, as shown in Table 7, the proposed leverage market microstructure model has the minimum DIC vales, which confirms that the leverage market microstructure model is superior to the leverage SV model for all three stock indices.

\section{Conclusion}

This paper is concerned with the specification for modeling financial leverage effect in the context of market microstructure model. With the new representations, it is easy to interpret the leverage effect in the model. The proposed model is validated by using simulated and real financial data series. A Bayesian Markov Chain Monte Carlo approach is developed for the generalized leverage market microstructure model. The results show that stock markets in China and the United States have obvious leverage effects, and the latter has stronger leverage effect than the former. Market microstructure model which allows for such an asymmetric property not only improves the ability to describe the dynamics of measurable 

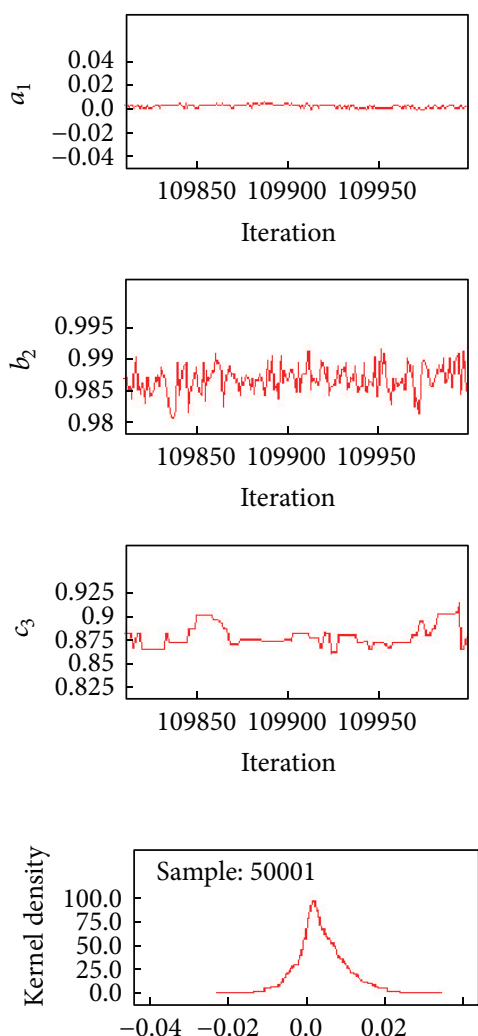

$a_{1}$
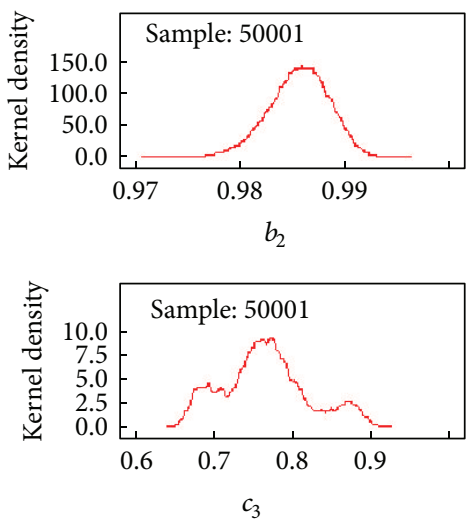
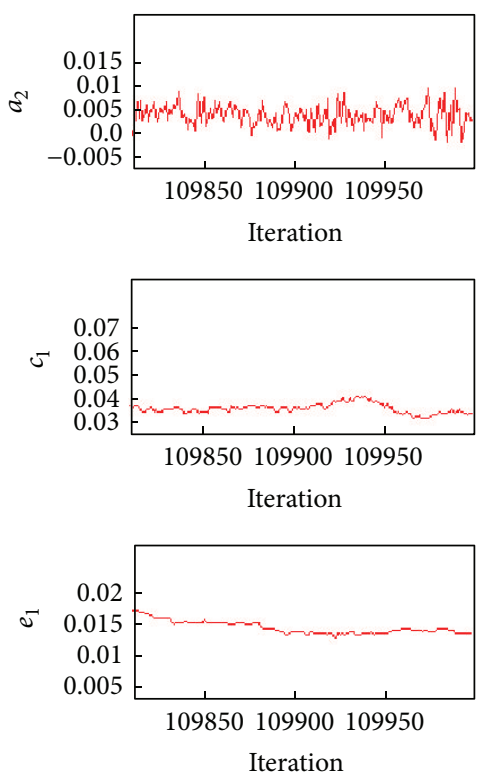

(a) Sample paths
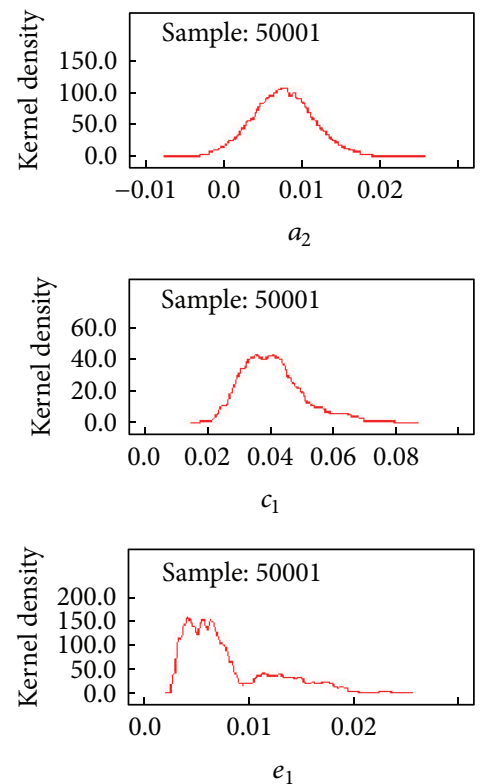

(b) Sample posterior densities
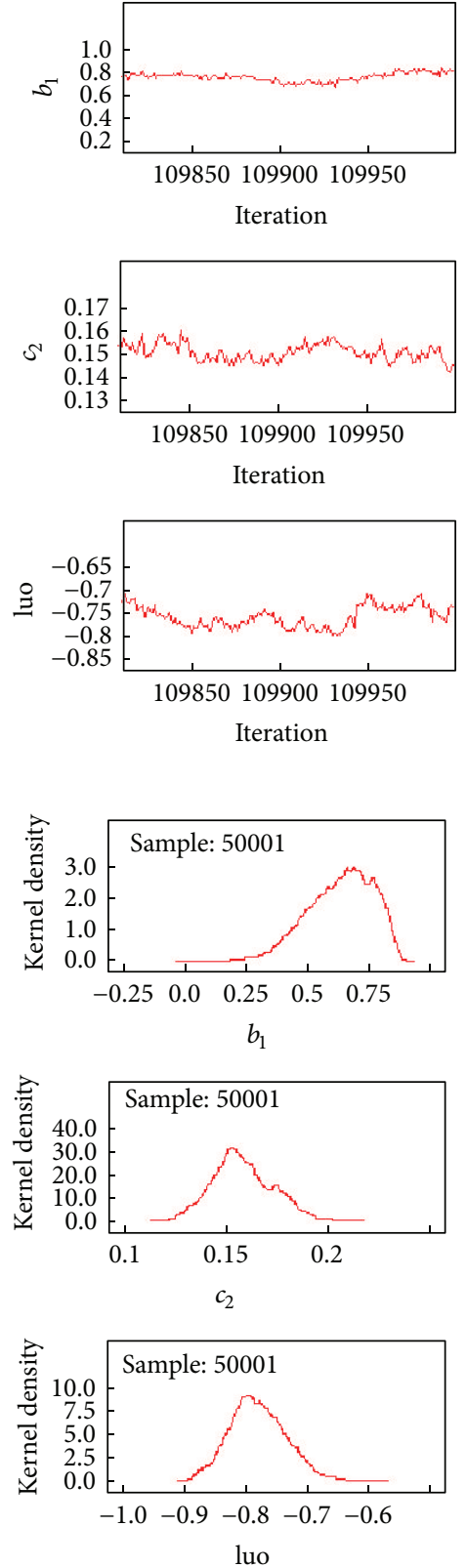

FIGURE 8: Sample path and density estimates for S\&P500.

market price but also provides more accurate price, which is important in practical application. Compared with the classical leverage SV model in terms of DIC, empirical results suggest evidence in favor of the proposed leverage market microstructure model.

\section{Appendices}

\section{A. The Proposal Density}

The most commonly used MCMC methods are the Metropolis-Hastings algorithm [36] and the Gibbs sampling algorithm [37].
The Gibbs sampling is a special case of the MetropolisHastings algorithm, which generates iterative samples from all the full conditional distributions. It can be justified by the Clifford-Hammersley theorem [38]. WinBUGS provides an easy and efficient implementation of the Gibbs sampling and has been widely used to estimate latent variables model. Also, it does not require the programmer to know the precise formulae for any prior density or likelihood. Its main strength lies in the ease with which any changes in the model, such as different autoregressive structures or polynomial state transitions, the choice of different prior distributions for the parameters, and the change from Gaussian to heavytailed observation error distributions. In this paper, we 
TABLE 6: Estimation results of the leverage SV model for three indices.

\begin{tabular}{|c|c|c|c|c|c|c|c|}
\hline Index & Parameter & Mean & $\mathrm{SD}$ & MC error & 2.5 percentile & Median & 97.5 percentile \\
\hline \multirow{4}{*}{$\begin{array}{l}\text { Shanghai } \\
\text { composite } \\
\text { index }\end{array}$} & $\mu$ & -4.366 & 0.1432 & 0.008468 & 4.583 & -4.38 & -3.998 \\
\hline & $\varphi$ & 0.9981 & 0.001106 & $1.803 E-5$ & 0.9956 & 0.9982 & 0.9997 \\
\hline & $\tau$ & 0.2792 & 0.02763 & 0.001367 & 0.2273 & 0.2785 & 0.3358 \\
\hline & $\rho$ & -0.3053 & 0.06265 & 0.002197 & -0.4257 & -0.3061 & -0.1794 \\
\hline \multirow{4}{*}{$\begin{array}{l}\text { Shenzhen } \\
\text { component } \\
\text { index }\end{array}$} & $\mu$ & -4.277 & 0.2668 & 0.01585 & -4.629 & -4.212 & -3.802 \\
\hline & $\varphi$ & 0.9985 & $9.161 E-4$ & $1.424 E-5$ & 0.9964 & 0.9986 & 0.9998 \\
\hline & $\tau$ & 0.239 & 0.02661 & 0.001095 & 0.3182 & 0.3677 & 0.4223 \\
\hline & $\rho$ & -0.2532 & 0.06992 & 0.002974 & -0.3839 & -0.2555 & -0.107 \\
\hline \multirow{4}{*}{ S\&P500 } & $\mu$ & -4.953 & 0.04702 & 0.003105 & -5.029 & -4.96 & -4.838 \\
\hline & $\varphi$ & 0.9987 & $5.776 E-4$ & $9.238 E-6$ & 0.9975 & 0.9988 & 0.9997 \\
\hline & $\tau$ & 0.1754 & 0.01317 & $7.608 E-4$ & 0.1494 & 0.1752 & 0.2029 \\
\hline & $\rho$ & -0.5942 & 0.05411 & 0.002845 & -0.6917 & -0.5972 & -0.4822 \\
\hline
\end{tabular}

TABLE 7: DIC vales of two models for three indices.

\begin{tabular}{llccc}
\hline Model & Index & $\bar{D}$ & $P_{D}$ & DIC \\
\hline & $\begin{array}{l}\text { Shanghai } \\
\text { composite index }\end{array}$ & -10944.0 & 285.6 & -10658.4 \\
\multirow{2}{*}{$\begin{array}{l}\text { The leverage SV } \\
\text { model }\end{array}$} & $\begin{array}{l}\text { Shenzhen } \\
\text { component index } \\
\end{array}$ & -10501.2 & 202.7 & -10298.5 \\
& S\&P500 & -17170.7 & 965.4 & -16205.3 \\
\hline $\begin{array}{l}\text { The leverage } \\
\text { market } \\
\text { microstructure } \\
\text { model }\end{array}$ & $\begin{array}{l}\text { Shanghai } \\
\text { composite index }\end{array}$ & -12703.9 & 1313.4 & -11390.5 \\
& $\begin{array}{l}\text { Shenzhen } \\
\text { component index }\end{array}$ & -13257.3 & 1560.0 & -11697.3 \\
\hline & S\&P500 & -18952.9 & 1936.0 & -17016.9 \\
\hline
\end{tabular}

use the freely available and user-friendly Bayesian software, WinBUGS, to do the single-move Gibbs sampling.

The Metropolis-Hastings sampling algorithm is appropriate for difficult full conditional distributions. In applying Metropolis-Hastings algorithms, it is necessary to choose the proposal density $q(\mathbf{y} \mid \mathbf{x})$. There are two common types of the proposal density. (i) In independence sampling, $q(\mathbf{y} \mid \mathbf{x})$ does not depend on $\mathbf{x}$; namely, $q(\mathbf{y} \mid \mathbf{x})=q(\mathbf{y})$. (ii) In the symmetric proposal density, $q(\mathbf{y} \mid \mathbf{x})=q(\mathbf{x} \mid \mathbf{y})$. An important special case of the symmetric Metropolis-Hastings method is the random-walk Metropolis algorithm, where $q(\mathbf{y} \mid \mathbf{x})=q(\mathbf{y}-\mathbf{x})$. A usual choice is to let $q(\mathbf{y} \mid \mathbf{x})$ be a symmetric Gaussian distribution centered at $\mathbf{x}$; namely, $\mathbf{y}=\mathbf{x}+\sigma \boldsymbol{\varepsilon}$, where $\boldsymbol{\varepsilon}$ is a random perturbation of Gaussian distribution and $\sigma$ is a scale parameter.

\section{B. The Posterior Density Distributions for the Parameters}

Consider the following

$$
\begin{aligned}
& p\left(\alpha_{1} \mid \alpha_{2}, \beta_{1}, \beta_{2}, \gamma_{1}, \gamma_{2}, \gamma_{3}, \rho, \phi, \sigma, P, Y\right) \\
& \quad \propto p\left(\phi \mid \alpha_{1}, \beta_{1}, \gamma_{1}\right) p\left(\alpha_{1}\right)=p\left(\phi_{0} \mid \alpha_{1}, \beta_{1}, \gamma_{1}\right)
\end{aligned}
$$

$$
\begin{aligned}
& \cdot \prod_{t=1}^{T} p\left(\phi_{k} \mid \phi_{k-1}, \alpha_{1}, \beta_{1}, \gamma_{1}\right) p\left(\alpha_{1}\right), \\
& p\left(\beta_{1} \mid \alpha_{1}, \alpha_{2}, \beta_{2}, \gamma_{1}, \gamma_{2}, \gamma_{3}, \rho, \phi, \sigma, P, Y\right) \\
& \propto p\left(\phi \mid \alpha_{1}, \beta_{1}, \gamma_{1}\right) p\left(\beta_{1}\right)=p\left(\phi_{0} \mid \alpha_{1}, \beta_{1}, \gamma_{1}\right) \\
& \cdot \prod_{t=1}^{T} p\left(\phi_{k} \mid \phi_{k-1}, \alpha_{1}, \beta_{1}, \gamma_{1}\right) p\left(\beta_{1}\right), \\
& p\left(\gamma_{1} \mid \alpha_{1}, \alpha_{2}, \beta_{1}, \beta_{2}, \gamma_{2}, \gamma_{3}, \rho, \phi, \sigma, P, Y\right) \\
& \propto p\left(\phi \mid \alpha_{1}, \beta_{1}, \gamma_{1}\right) p\left(\gamma_{1}\right)=p\left(\phi_{0} \mid \alpha_{1}, \beta_{1}, \gamma_{1}\right) \\
& \cdot \prod_{t=1}^{T} p\left(\phi_{k} \mid \phi_{k-1}, \alpha_{1}, \beta_{1}, \gamma_{1}\right) p\left(\gamma_{1}\right), \\
& p\left(\alpha_{2} \mid \alpha_{1}, \beta_{1}, \beta_{2}, \gamma_{1}, \gamma_{2}, \gamma_{3}, \rho, \phi, \sigma, P, Y\right) \\
& \propto p\left(\sigma \mid \alpha_{2}, \beta_{2}, \gamma_{2}\right) p\left(\alpha_{2}\right)=p\left(\sigma_{0} \mid \alpha_{2}, \beta_{2}, \gamma_{2}\right) \\
& \cdot \prod_{t=1}^{T} p\left(\sigma_{k} \mid \sigma_{k-1}, \alpha_{2}, \beta_{2}, \gamma_{2}\right) p\left(\alpha_{2}\right), \\
& p\left(\beta_{2} \mid \alpha_{1}, \beta_{1}, \alpha_{2}, \gamma_{1}, \gamma_{2}, \gamma_{3}, \rho, \phi, \sigma, P, Y\right) \\
& \propto p\left(\sigma \mid \alpha_{2}, \beta_{2}, \gamma_{2}\right) p\left(\beta_{2}\right)=p\left(\sigma_{0} \mid \alpha_{2}, \beta_{2}, \gamma_{2}\right) \\
& \cdot \prod_{t=1}^{T} p\left(\sigma_{k} \mid \sigma_{k-1}, \alpha_{2}, \beta_{2}, \gamma_{2}\right) p\left(\beta_{2}\right), \\
& p\left(\gamma_{2} \mid \alpha_{1}, \beta_{1}, \alpha_{2}, \beta_{2}, \gamma_{1}, \gamma_{3}, \rho, \phi, \sigma, P, Y\right) \\
& \propto p\left(\sigma \mid \alpha_{2}, \beta_{2}, \gamma_{2}\right) p\left(\gamma_{2}\right)=p\left(\sigma_{0} \mid \alpha_{2}, \beta_{2}, \gamma_{2}\right) \\
& \cdot \prod_{t=1}^{T} p\left(\sigma_{k} \mid \sigma_{k-1}, \alpha_{2}, \beta_{2}, \gamma_{2}\right) p\left(\gamma_{2}\right), \\
& p\left(\gamma_{3} \mid \alpha_{1}, \beta_{1}, \alpha_{2}, \beta_{2}, \gamma_{1}, \gamma_{2}, \rho, \phi, \sigma, P, Y\right)
\end{aligned}
$$




$$
\begin{aligned}
& \propto p\left(P \mid \phi, \sigma, \gamma_{3}\right) p\left(\gamma_{3}\right)=p\left(P_{0} \mid \gamma_{3}\right) \\
& \cdot \prod_{t=1}^{T} p\left(P_{k} \mid P_{k-1}, \phi_{k-1}, \sigma_{k-1}, \gamma_{3}\right) p\left(\gamma_{3}\right) .
\end{aligned}
$$

\section{Conflict of Interests}

The authors declare that there is no conflict of interests regarding the publication of this paper.

\section{Acknowledgments}

This work was supported by the National Natural Science Foundation for Young Scientists of China (51507015, 61403045), the National Natural Science Foundation of China (71271215, 61540037), and the Natural Science Foundation of Hunan Province, China (2015JJ3008).

\section{References}

[1] F. Black, "Studies of stock market volatility changes," in Proceedings of the American Statistical Association: Business and Economic Statistics Section, pp. 177-181, American Statistical Association, Alexandria, Va, USA, 1976.

[2] A. A. Christie, "The stochastic behavior of common stock variances: value, leverage and interest rate effects," Journal of Financial Economics, vol. 10, no. 4, pp. 407-432, 1982.

[3] T. Bollerslev, "Generalized autoregressive conditional heteroskedasticity," Journal of Econometrics, vol. 31, no. 3, pp. 307$327,1986$.

[4] D. B. Nelson, "Conditional heteroskedasticity in asset returns: a new approach," Econometrica, vol. 59, no. 2, pp. 347-370, 1991.

[5] L. R. Glosten, R. Jagannathan, and D. E. Runkle, "On the relation between the expected value and the volatility of the nominal excess return on stocks," The Journal of Finance, vol. 48, no. 5, pp. 1779-1801, 1993.

[6] A. C. Harvey and N. Shephard, "The estimation of an asymmetric stochastic volatility model for asset returns," Journal of Business and Economic Statistics, vol. 14, no. 4, pp. 429-434, 1996.

[7] E. Jacquier, N. G. Polson, and P. E. Rossi, "Bayesian analysis of stochastic volatility models with fat-tails and correlated errors," Journal of Econometrics, vol. 122, no. 1, pp. 185-212, 2004.

[8] J. Yu, "MCMC methods for estimating stochastic volatility models with leverage effects: comment on Jacquier, Polson and Rossi (2002)," Economics Working Papers, 2002.

[9] J. Yu, "On leverage in a stochastic volatility model," Journal of Econometrics, vol. 127, no. 2, pp. 165-178, 2005.

[10] Y. Omori, S. Chib, N. Shephard, and J. Nakajima, "Stochastic volatility with leverage: fast and efficient likelihood inference," Journal of Econometrics, vol. 140, no. 2, pp. 425-449, 2007.

[11] J. J. J. Wang, J. S. K. Chan, and S. T. B. Choy, "Stochastic volatility models with leverage and heavy-tailed distributions: a Bayesian approach using scale mixtures," Computational Statistics \& Data Analysis, vol. 55, no. 1, pp. 852-862, 2011.

[12] M. Asai and M. McAleer, "Alternative asymmetric stochastic volatility models," Econometric Reviews, vol. 30, no. 5, pp. 548564, 2011.
[13] G. Tsiotas, "On generalised asymmetric stochastic volatility models," Computational Statistics \& Data Analysis, vol. 56, no. 1, pp. 151-172, 2012.

[14] M. Iino and T. Ozaki, "A nonlinear model for financial dynamics," in Proceeding of the International Symposium on Frontiers of Time Series Modeling, pp. 7-9, Tokyo, Japan, February 2000.

[15] M. O'Hara, Market Microstructure Theory, Basil Blackwell, Oxford, UK, 1995.

[16] A. Marshall, Principles of Economics, Macmillan and Company, London, UK, 1890.

[17] Y. C. Zhang, "Toward a theory of marginally efficient markets," Physica A: Statistical Mechanics and Its Applications, vol. 269, no. 1, pp. 30-44, 1999.

[18] Y. Amihud and H. Mendelson, "Asset pricing and the bid-ask spread," Journal of Financial Economics, vol. 17, no. 2, pp. 223249, 1986.

[19] M. R. Reinganum, "Market microstructure and asset pricing: an empirical investigation of NYSE and NASDAQ securities," Journal of Financial Economics, vol. 28, no. 1-2, pp. 127-147, 1990.

[20] H. Peng, T. Ozaki, and V. Haggan-Ozaki, "Modeling and asset allocation for financial markets based on a discrete time microstructure model," The European Physical Journal B, vol. 31, no. 2, pp. 285-293, 2003.

[21] H. Peng, Y. Tamura, W. Gui, and T. Ozaki, "Modelling and asset allocation for financial markets based on a stochastic volatility microstructure model," International Journal of Systems Science, vol. 36, no. 6, pp. 315-327, 2005.

[22] H. Peng, G. Kitagawa, Y. Tamura, Y. Tanokura, M. Gan, and X. H. Chen, "Detection of low-frequency large-amplitude jump in financial time series," in Proceedings of the 46th IEEE Conference on Decision and Control (CDC '07), pp. 4944-4949, IEEE, New Orleans, La, USA, December 2007.

[23] H. Peng, G. Kitagawa, Y. Tamura, Y. H. Xi, Y. M. Qin, and X. H. Chen, "A modeling approach to financial time series based on market microstructure model with jumps," Applied Soft Computing Journal, vol. 29, pp. 40-51, 2015.

[24] Y. H. Xi, H. Peng, and C. Ruan, "Numerical solution for market microstructure model with nonhomogeneous Poisson jump," International Journal of Digital Content Technology and Its Applications, vol. 6, no. 23, pp. 560-568, 2012.

[25] Y. H. Xi, H. Peng, Y. M. Qin, W. B. Xie, and X. H. Chen, "Bayesian analysis of heavy-tailed market microstructure model and its application in stock markets," Mathematics and Computers in Simulation, vol. 117, pp. 141-153, 2015.

[26] J. P. Bouchaud and R. A. Cont, "A Langevin approach to stock market fluctuations and crashes," The European Physical Journal $B$, vol. 6, no. 4, pp. 543-550, 1998.

[27] D. J. Higham, "An algorithmic introduction to numerical simulation of stochastic differential equations," Society for Industrial and Applied Mathematics, vol. 43, no. 3, pp. 525-546, 2001.

[28] E. Jacquier, N. G. Polson, and P. E. Rossi, "Bayesian analysis of stochastic volatility models," Journal of Business and Economic Statistics, vol. 20, no. 1, pp. 69-87, 2002.

[29] S. Kim, N. Shephard, and S. Chib, "Stochastic volatility: likelihood inference and comparison with ARCH models," Review of Economic Studies, vol. 65, no. 3, pp. 361-393, 1998.

[30] S. Chib, F. Nardari, and N. Shephard, "Markov chain Monte Carlo methods for stochastic volatility models," Journal of Econometrics, vol. 108, no. 2, pp. 281-316, 2002.

[31] J. Nakajima and Y. Omori, "Leverage, heavy-tails and correlated jumps in stochastic volatility models," Computational Statistics \& Data Analysis, vol. 53, no. 6, pp. 2335-2353, 2009. 
[32] L. Bauwens and M. Lubrano, "Bayesian inference on GARCH models using the Gibbs sampler," The Econometrics Journal, vol. 1, no. 1, pp. c23-c46, 1998.

[33] I. D. Vrontos, P. Dellaportas, and D. N. Politis, "Full bayesian inference for GARCH and EGARCH models," Journal of Business and Economic Statistics, vol. 18, no. 2, pp. 187-198, 2000.

[34] T. Nakatsuma, "Bayesian analysis of ARMA-GARCH models: a Markov chain sampling approach," Journal of Econometrics, vol. 95, no. 1, pp. 57-69, 2000.

[35] R. Meyer and J. Yu, "BUGS for a Bayesian analysis of stochastic volatility models," The Econometrics Journal, vol. 3, no. 2, pp. 198-215, 2000.

[36] W. K. Hastings, "Monte Carlo sampling methods using Markov chains and their applications," Biometrika, vol. 57, no. 1, pp. 97$109,1970$.

[37] S. Geman and D. Geman, "Stochastic relaxation, Gibbs distributions, and the Bayesian restoration of images," IEEE Transactions on Pattern Analysis and Machine Intelligence, vol. 6, no. 6, pp. 721-741, 1984.

[38] P. Clifford and J. Hammersley, "Markov fields on finite graphs and lattices," Working Paper, Oxford University, 1970. 


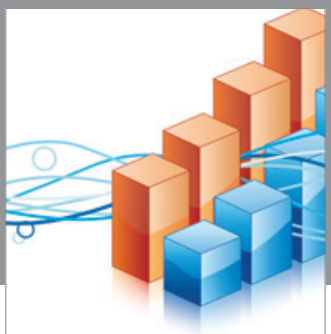

Advances in

Operations Research

vatem alat4

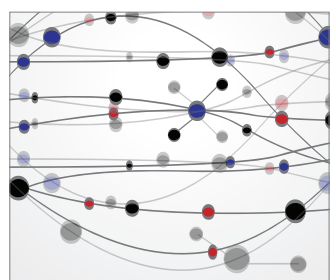

\section{The Scientific} World Journal
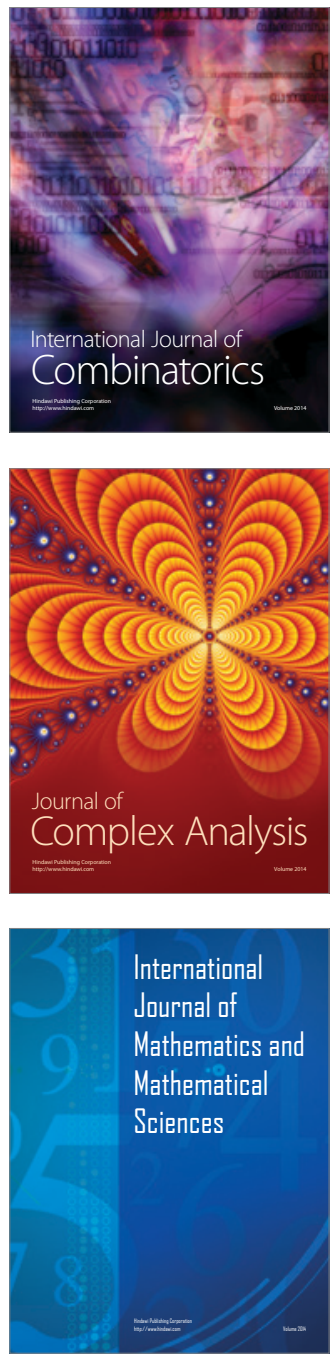
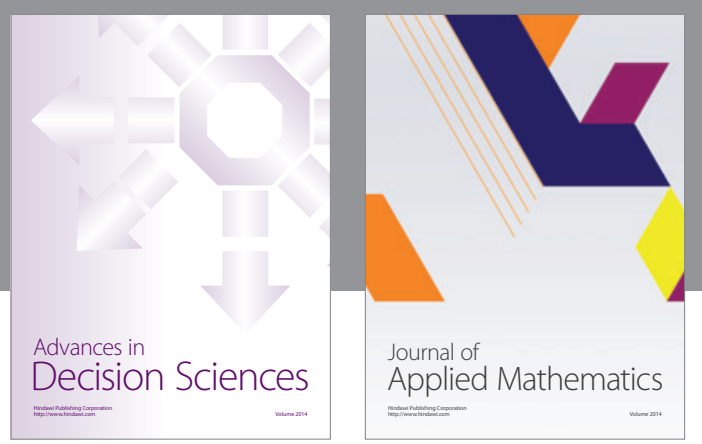

Algebra

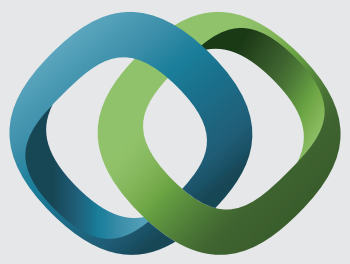

\section{Hindawi}

Submit your manuscripts at

http://www.hindawi.com
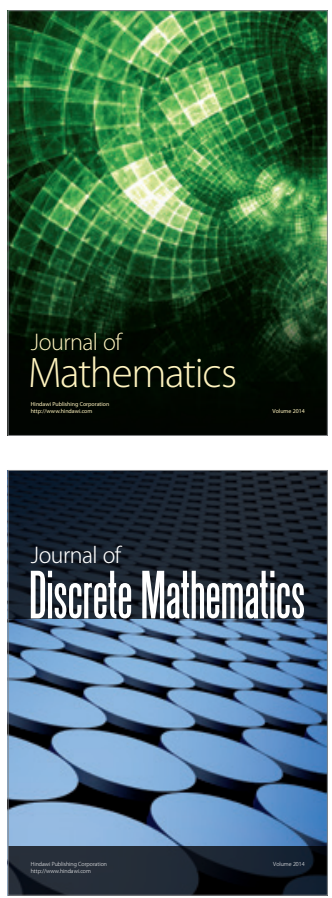

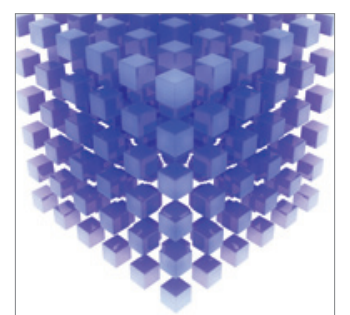

Mathematical Problems in Engineering
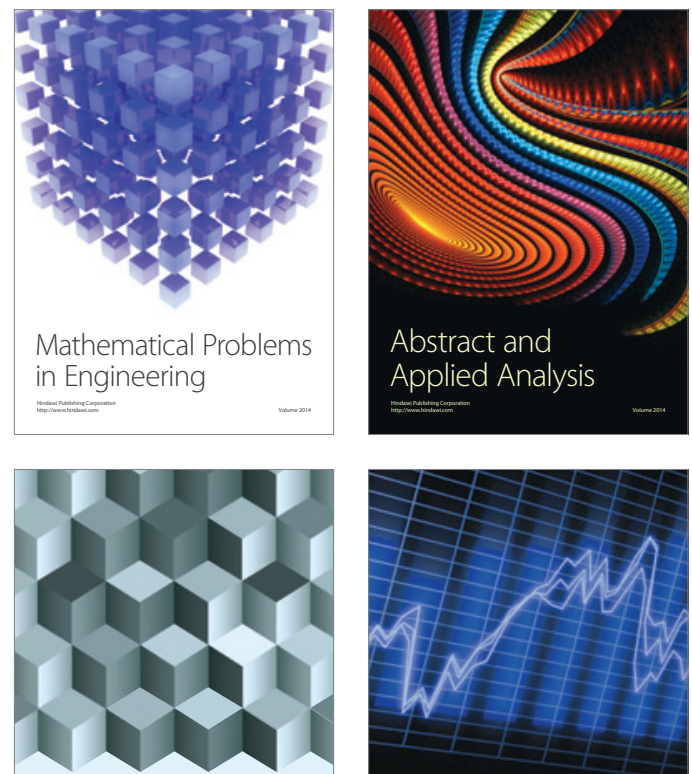

Journal of

Function Spaces

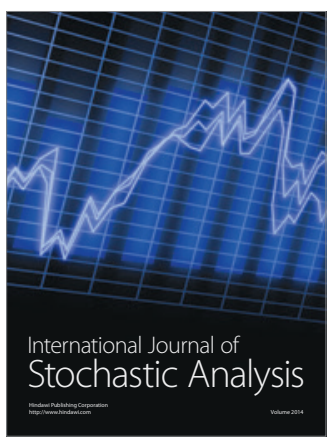

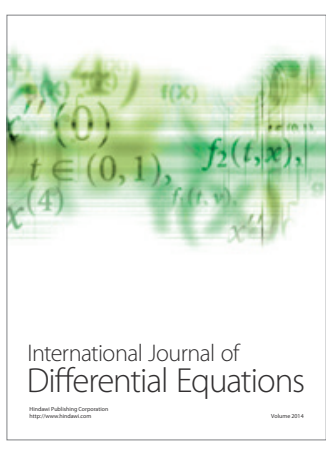
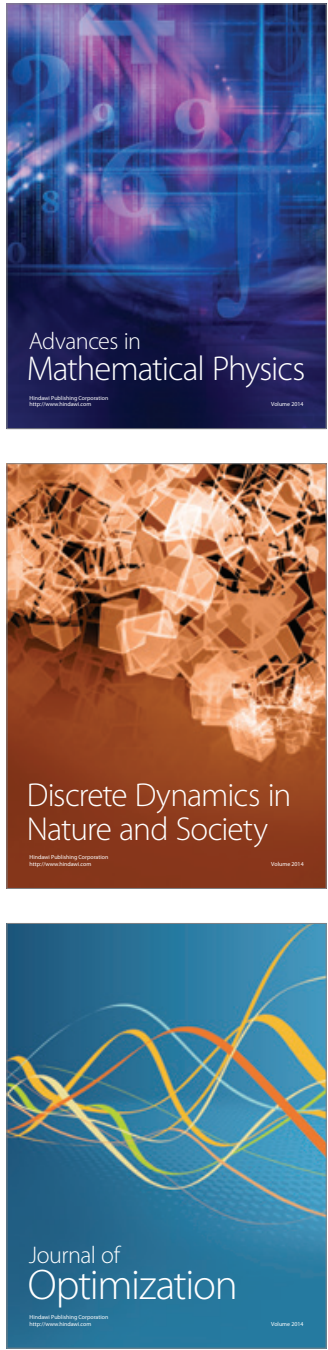